\title{
ON ELASTIC SOLIDS WITH LIMITING SMALL STRAIN: MODELLING AND ANALYSIS
}

\author{
MIROSLAV BULÍČEK, JOSEF MÁLEK, K. R. RAJAGOPAL, AND ENDRE SÜLI
}

\begin{abstract}
In order to understand nonlinear responses of materials to external stimuli of different sort, be they of mechanical, thermal, electrical, magnetic, or of optical nature, it is useful to have at one's disposal a broad spectrum of models that have the capacity to describe in mathematical terms a wide range of material behavior. It is advantageous if such a framework stems from a simple and elegant general idea. Implicit constitutive theory of materials provides such a framework: while being built upon simple ideas, it is able to capture experimental observations with the minimum number of physical quantities involved. It also provides theoretical justification in the full three-dimensional setting for various models that were previously proposed in an ad hoc manner. From the perspective of the theory of nonlinear partial differential equations, implicit constitutive theory leads to new classes of challenging mathematical problems. This study focuses on implicit constitutive models for elastic solids in general, and on its subclass consisting of elastic solids with limiting small strain. After introducing the basic concepts of implicit constitutive theory, we provide an overview of results concerning modeling within the framework of continuum mechanics. We then concentrate on the mathematical analysis of relevant boundary-value problems associated with models with limiting small strain, and we present the first analytical result concerning the existence of weak solutions in general three-dimensional domains.
\end{abstract}

\section{INTRODUCTION}

An elastic (nondissipative) response of a material is an idealization that corresponds to a certain perfect (ideal) situation. Foucault's pendulum will swing for several months before the observer will notice a slight decrease in the amplitude of its oscillation, until finally dissipation due to friction of the air will bring the pendulum to rest, its equilibrium state. The motion of Foucault's pendulum is thus usually described as a conservative vibrating system, which is however merely an approximation of a dissipative system in which the dissipation is negligible. The approximation that a body is elastic is made in a similar spirit wherein a dissipative body, which undergoes negligible dissipation in all processes that it is subjected to, is approximated as a conservative system. While elasticity is useful in describing certain deformations in solid materials, particularly those in which the solid stores almost all of its energy with hardly any of it being dissipated, it is fair to say that improved understanding of the modeling of elastic responses of bodies can have significant consequences on the modeling of complex materials where elastic deformation represents merely a part of the complete deformation process. As examples, one can think of viscoelastic materials, i.e., materials that emulate the behavior typical of both solids and fluids; or electro- or magneto-elastic materials where the combination of mechanical and electrical or magnetic effects are important; or fluid structure interaction problems, that can be viewed as multi-constituent problems, with a solid and a fluid constituent, which, though separated, share a common interface; or indeed thermo-elastic materials. For each of these, the understanding of the system as a whole is crucial.

Key words and phrases. implicit constitutive theory, elastic solid, small strain, nonlinear model, partial differential equation (PDE), boundary-value problem, weak solution, existence theory, qualitative results.

The authors acknowledge the support of the ERC-CZ project LL1202 financed MŠMT. E. Süli was also supported by the EPSRC Science and Innovation award to the Oxford Centre for Nonlinear PDE (EP/E035027/1). M. Bulíček is also thankful to the Neuron Fund for Support of Science for its support. M. Bulíček is a researcher in the University Centre for Mathematical Modelling, Applied Analysis and Computational Mathematics (Math MAC). M. Bulíček and J. Málek are researchers of the Nečas Center for Mathematical Modeling (NCMM). 
Just as a painter needs to have a palette containing all of the basic colours so as to be able to express himself, any modeler will wish to have a rich enough repertoire of models so as to adequately describe all phenomena of interest. The recently established theory of implicit constitutive relations (see [46, 48]) provides such a robust repertoire of models. The theory is built upon simple ideas and has proved itself useful in capturing experimental observations with a minimum number of physical quantities involved; it has furthermore provided theoretical justification for several models that were perviously proposed in a rather ad hoc manner.

In this study, we focus on the behaviour of elastic (nondissipative) solids that are best described by implicit constitutive relations. We shall illustrate how the theory of implicit constitutive relations extends the class of models that were employed earlier, even though the number of physical quantities involved in implicit constitutive models is the same as in classical linear models. Following [49] we shall explain how one can easily justify nonlinear elastic models involving the linearized (small) strain. In particular, the setting of solids provides a firm theoretical background for models with limiting (large or small) strains. It is thus possible to have models in which the linearized strain is in all circumstances a bounded function, even when the stress is very large. This class of implicit constitutive models, developed by Rajagopal in [48], and which are referred to as limiting strain models, has the potential to be useful in describing the behavior of brittle materials near crack tips or notches, or concentrated loads inside the body or on its boundary. Both of these effects lead to stress concentration even though the gradient of the displacement is small.

The aim of this survey is at least threefold. First, we summarize the available literature concerning implicitly constituted elastic solids and highlight some of the important features of the theory. We then focus on the subclass of strain-limiting models and collect relevant available results concerning modeling, PDE analysis, the solution of reduced problems and computer simulations. Finally, after formulating a typical boundary-value problem for a strain-limiting model, we present the first existence result for the associated system of nonlinear partial differential equations in a general three-dimensional domain.

It is worth mentioning that materials that can be characterized by implicit constitutive relations appear naturally in many other areas that are beyond the scope of this paper (e.g. in flows through porous media, non-Newtonian fluid mechanics, models of viscoelastic solids, and in the enforcement of interface and boundary conditions).

The paper is structured as follows. In the next section, we start by discussing constitutive relations for vibrating mass parameter systems in order to motivate the framework of implicit constitutive relations for describing both solids and fluids. Then, still in Section 2, we recall the balance equations of continuum mechanics and discuss several classes of constitutive relations that are in place, ranging from linear to nonlinear, and from explicit to implicit. In Section 3 we focus our attention on elastic solids that are described by implicit constitutive relations, particularly those that exhibit a limiting strain. In the course of highlighting the importance of this class of models, we shall discuss both mechanical and thermomechanical issues. We shall survey the results established in the literature to date in the directions of: solving semi-inverse problems; the PDE analysis of relevant boundary-value problems (BVP) such as the anti-plane stress problem and the spatially periodic problem; as well as computational results and asymptotic analysis. Several results concerning the PDE analysis of BVP problems, established for these models in previous papers, are stated in the form of theorems in the first subsection of Section 4. In the rest of Section 4 we present new results concerning the analysis of a typical BVP for a limiting small strain model in a general bounded three-dimensional domain. Since this is a survey article, our intention has not been to obtain the most general analytical result, but rather to illustrate specific mathematical features of the model. Specifically, we study the homogeneous Dirichlet problem in the static case and present new results concerning the existence of a weak solution, its uniqueness and regularity. The mathematical analysis of evolutionary limiting strain models, problems with specified traction on the boundary of the domain, the mathematical analysis of numerical approximations to these models (besides [9]) and careful computational studies are left as future challenges. 


\section{IMPLICITLY CONSTITUTED MATERIALS}

Vibrating lumped parameter systems. Before discussing implicit constitutive theories within the context of continua, it is worth considering briefly the possibility of implicit equations to describe the response of a vibrating lumped parameter system and of a mass-spring system. When considering simple vibrating systems as represented in Figs. 1, 2 or 3, one invariably describes the response of the spring and the dashpot by providing an expression for the forces that are developed in the spring and the dashpot with respect to the displacement and velocity, respectively; that is, one provides expressions for the spring force $f_{s}$ and the dashpot force $f_{d}$ as

$$
f_{s}=g(x) \quad \text { and } \quad f_{d}=h(\dot{x})
$$

where $x$ and $\dot{x}$ are the displacement and the velocity, respectively.
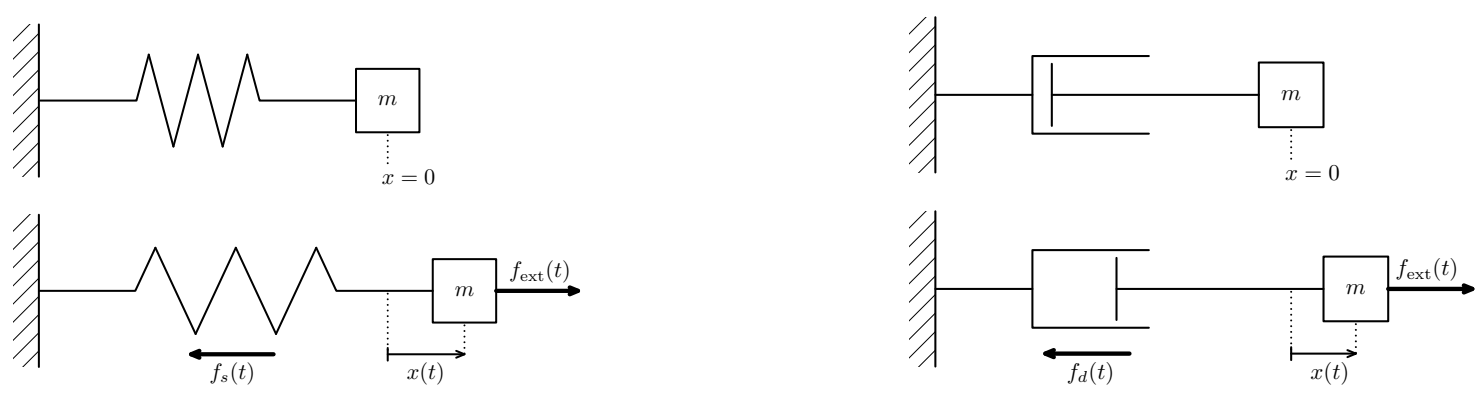

FIGURE 1. Mass-spring and mass-dashpot systems. The figures at the top depict a mass-spring system and a mass-dashpot system in their equilibrium positions. When applying an external force of magnitude $f_{\text {ext }}$ in the $x$-direction the mass-spring system gets into motion; there is a spring force acting in the opposite direction to the applied external force and having magnitude $f_{s}$, pushing the spring into its equilibrium position. As the outcome of these forces, the mass is placed at the position $x(t)$ at time $t$ (see the figure at the bottom left corner). Similarly, when applying an external force of magnitude $f_{\text {ext }}$ in the $x$-direction, the massdashpot system gets into motion and takes up the position $x(t)$ as a result of two forces: the external force and the force due to friction of the fluid in the dashpot; the latter force acts in the opposite direction to the applied external force and has magnitude $f_{d}$; see the figure at the bottom right corner.

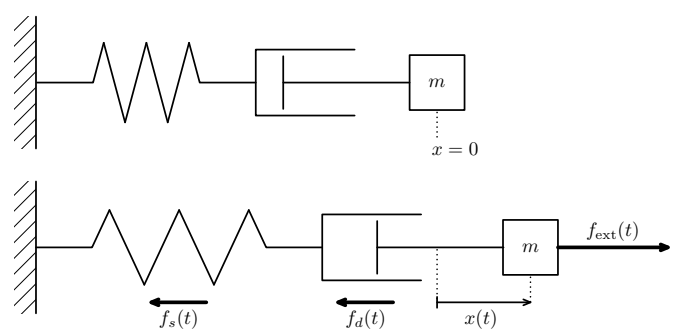

FIGURE 2. Mass-spring-dashpot system. The figure at the top depicts a mass-springdashpot system in its equilibrium position. When applying an external force of magnitude $f_{\text {ext }}$ in the $x$-direction, the system takes on the position $x(t)$ at time $t$ as a result of the relevant forces.

In the case of a linear spring and a linear dashpot, we prescribe

$$
f_{s}=k x \quad \text { and } \quad f_{d}=c \dot{x},
$$

where $k$ is the spring constant and $c$ the constant for the dashpot. The balance of linear momentum (i.e., the equation of the motion of classical particle mechanics) then has the form

$$
\frac{d(m \boldsymbol{v})}{d t}=\boldsymbol{f},
$$


where $\boldsymbol{f}$ stands for all relevant forces acting on the particle having mass $m$ and located at the position $\boldsymbol{x}=(x, y, z)$, with velocity $\boldsymbol{v}=(\dot{x}, \dot{y}, \dot{z})$. In our one-dimensional setting, (3) simplifies to the form

$$
m \ddot{x}+f_{s}+f_{d}=f_{\text {ext }},
$$

where $f_{\text {ext }}$ is the given external force (acting merely in the $x$-direction), and $m$ is the mass of the object. Equations (1) and (2) are essentially the constitutive specifications for the spring and the dashpot. On inserting (1) into (4), one obtains an ordinary differential equation for the displacement $x$ and this equation has been studied in great detail for a variety of nonlinear functions $g$ and $h$.

Recently, Rajagopal [50] has articulated the need for implicit relationships between the force and the displacement/velocity for the spring/dashpot system. That one cannot specify a forcedisplacement relation for the spring becomes obvious if the relationship is that portrayed in Fig. 3. Such a response corresponds to a spring placed in parallel with an inextensible string of fixed length $L$, say, as also sketched in Fig. 3. Similarly, one cannot specify a force-velocity relation corresponding to a Bingham-like dashpot as drawn in Fig. 4. In this case it is much more sensible to prescribe the velocity $\dot{x}$ in terms of the dashpot force $f_{d}$. In general, one cannot explicitly prescribe the appropriate kinematical quantity in terms of the force and one might only be able to specify an implicit relations of the form

$$
g\left(f_{s}, x\right)=0 \quad \text { and } \quad g\left(f_{d}, \dot{x}\right)=0 .
$$

More general implicit relationships between the forces and kinematical quantities are possible but we shall not discuss them here; the interested reader is referred to [50].
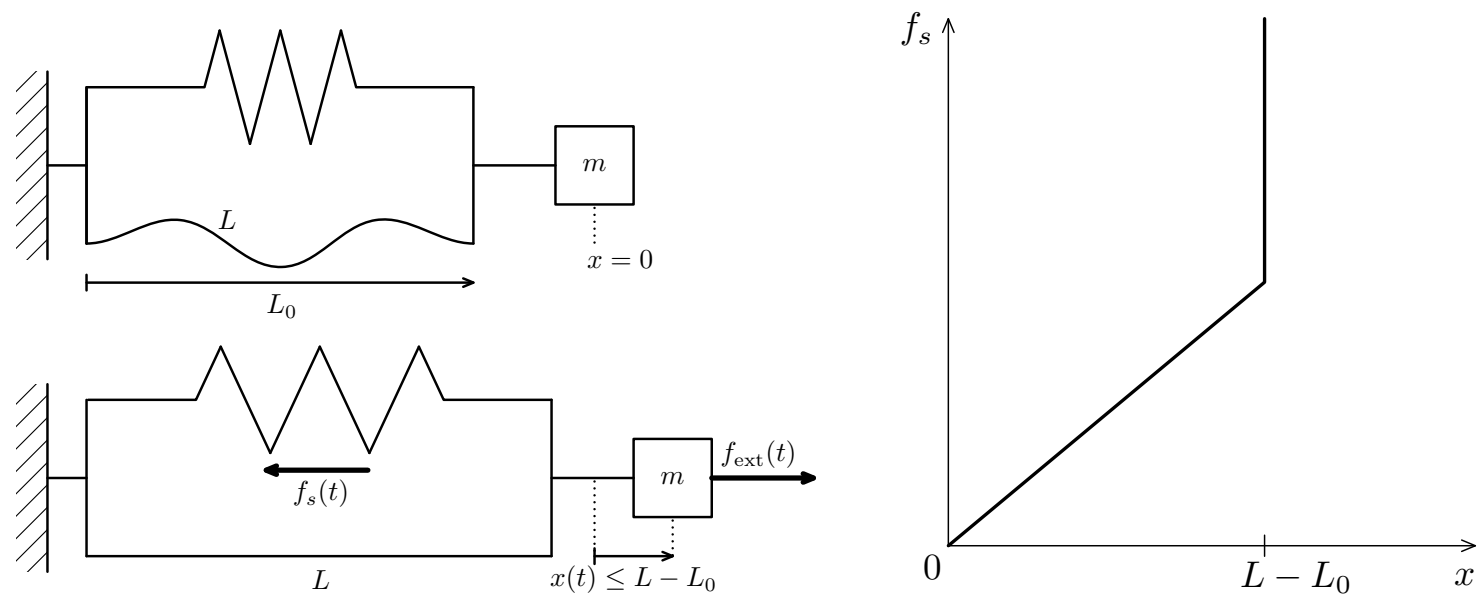

FIGURE 3. Mass-spring-wire system. The figure at the top (left) depicts a mass-springwire system in its equilibrium position. The wire of the maximal length $L$ cannot break whatever force is applied to it. When applying an external force of magnitude $f_{\text {ext }}$ in the $x$-direction the system gets into motion, but the extension of the spring is limited by the maximal length of the wire. Once the maximal length of the wire is attained, no change in the mass position occurs with increasing $f_{\text {ext }}$ and consequently $f_{s}$. The corresponding response is depicted in the figure on the right.

Let us consider the simpler subcase of the relation (5) when the spring and dashpot are described respectively through

$$
x=\varphi\left(f_{s}\right) \quad \text { and } \quad \dot{x}=\psi\left(f_{d}\right) .
$$

We notice that the responses drawn in Figs. 3 and 4 cannot be described by the second equation in (1) but can be described by the second equation in (6). However, now we cannot substitute (6) into (4) to obtain a single equation for the displacement. One has to solve the system of equations 


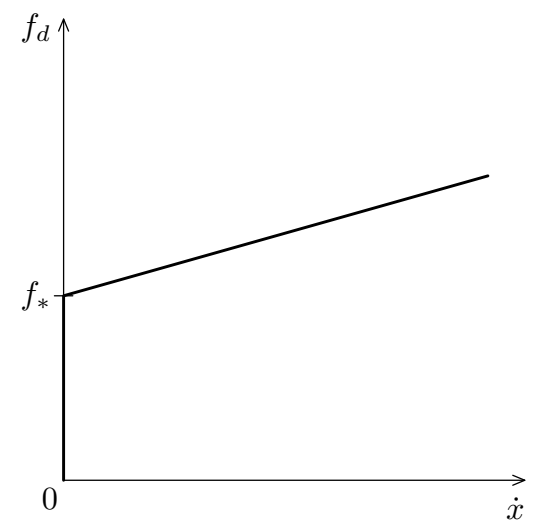

FIGURE 4. The response of a Bingham like mass-dashpot system. The constant $f_{*}$ denotes the threshold force and the dashpot velocity is zero until the threshold force is exceeded.

(1) and (6) simultaneously. Recently, Darbha, Nakshatrala and Rajagopal [19] have solved such systems.

In the more complicated case of the system of equations (4) and (5) we once again have a system of three equations for the three unknowns $x, f_{s}$ and $f_{d}$. The problem is quite challenging as the implicit relations (5) may not allow one to express the kinematics in terms of the force or the force in terms of kinematics (see Pražák and Rajagopal [44] for a discussion of the relevant issues).

Turning our attention to the description of continua, we note a similar development with regard to constitutive specifications.

Balance equations in continuum mechanics. The fundamental equations that form the basis of continuum mechanics stem from balancing mass and linear and angular momenta over any subpart of the body. These equations differ depending on the state of the body with which these balance equations are associated. In Fig. 5, where the concept of motion $\chi$ is depicted, leading then naturally to the notion of velocity $\boldsymbol{v}$ and deformation gradient $\mathbf{F}$, defined as

$$
\boldsymbol{v}:=\frac{\partial \boldsymbol{\chi}}{\partial t} \quad \text { and } \quad \mathbf{F}:=\frac{\partial \boldsymbol{\chi}}{\partial \boldsymbol{X}}
$$

the states of the body are its reference (initial) configuration $\Omega_{R}$ and its current configuration $\Omega$.

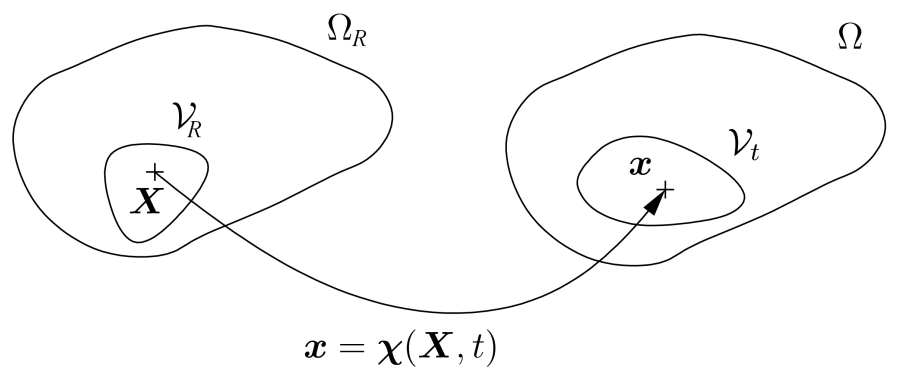

FIGURE 5. Reference and current configurations of the body. 
In the current configuration of the body, i.e., in the set $\Omega$, the balance equations for mass, linear momentum and angular momentum ${ }^{1}$ take the form

$$
\begin{aligned}
\frac{\partial \varrho}{\partial t}+\operatorname{div}_{x}(\varrho \boldsymbol{v}) & =0 \\
\frac{\partial(\varrho \boldsymbol{v})}{\partial t}+\operatorname{div}_{x}(\varrho \boldsymbol{v} \otimes \boldsymbol{v}) & =\operatorname{div}_{x} \mathbf{T}+\varrho \boldsymbol{f}, \\
\mathbf{T} & =\mathbf{T}^{T}
\end{aligned}
$$

where $\varrho$ is the density of the material and the symmetric tensor $\mathbf{T}$ is the Cauchy stress. While these equations have general validity, they are not sufficient to determine the stress in the body and the displacement/velocity of the body. In order to obtain a closed system of equations, we need to know how the body is constituted, and this information is provided by the constitutive relation for the body, which relates the Cauchy stress $\mathbf{T}$ (or any other appropriate measure of stress) and the kinematical variables that are relevant to the response. When a body that responds to other fields such as a thermal, magnetic or electric field, equation (8) has to be augmented by the energy equation and Maxwell's equations and one needs additional constitutive relations for the heat flux, radiant energy, and any other appropriate field variable. Any such constitutive relation specifies a class of materials. Thus, by material we mean, in what follows, a constitutive relation characterizing a certain (idealized) response of the real material in a particular class of deformation processes. The same convention regarding nomenclature concerns the words fluid and solid.

The above balance equations, when expressed with respect to the reference configuration, i.e., in the set $\Omega_{R}$, take the form

$$
\begin{aligned}
\varrho & =\varrho_{R} \operatorname{det} \mathbf{F}, \\
\varrho_{R} \frac{\partial^{2} \chi}{\partial t^{2}} & =\operatorname{div}_{X} \mathbf{S}+\varrho_{R} \boldsymbol{f}, \\
\mathbf{S F}^{T} & =\mathbf{F S}^{T},
\end{aligned}
$$

where $\varrho_{R}$ is the reference density and $\mathbf{S}$ denotes the first Piola-Kirchhoff stress tensor

$$
\mathbf{S}:=(\operatorname{det} \mathbf{F}) \mathbf{T F}^{-T} \text {. }
$$

We complete this part of the exposition by recalling various quantities, which are based on $\boldsymbol{F}$ and $\boldsymbol{v}$ and which measure the deformation and the rate of deformation respectively. These quantities will be finally used to relate the Cauchy stress tensor $\mathbf{T}$ with the kinematic quantities and to complete the system of equations (8) and (9) respectively. First, the displacement $\boldsymbol{u}$ is defined as

$$
\boldsymbol{u}:=\chi(\boldsymbol{X}, t)-\boldsymbol{X} .
$$

Then, the left and the right Cauchy-Green stretch tensors $\mathbf{B}$ and $\mathbf{C}$ and the Green-St. Venant strain $\mathbf{E}$ are given through

$$
\mathbf{B}:=\mathbf{F F}^{T}, \quad \mathbf{C}:=\mathbf{F}^{T} \mathbf{F} \quad \text { and } \quad \mathbf{E}:=\frac{1}{2}(\mathbf{C}-\mathbf{I}) .
$$

The stretch tensors $\mathbf{U}$ and $\mathbf{V}$ that appear through the application of the Polar Decomposition Theorem ${ }^{2}$ to $\mathbf{F}$, are related to the tensors $\mathbf{B}$ and $\mathbf{C}$ through

$$
\mathbf{V}^{2}=\mathbf{B} \quad \text { and } \quad \mathbf{U}^{2}=\mathbf{C} .
$$

The velocity gradient, denoted either by $\mathbf{L}$ or by $\nabla \boldsymbol{v}$, can be split into its symmetric part $\mathbf{D}$ (or $\mathbf{D} \boldsymbol{v})$ and its antisymmetric part $\mathbf{W}$, i.e.,

$$
\mathbf{D}:=\frac{1}{2}\left(\mathbf{L}+\mathbf{L}^{T}\right) \quad \text { and } \quad \mathbf{W}:=\frac{1}{2}\left(\mathbf{L}-\mathbf{L}^{T}\right) .
$$

\footnotetext{
${ }^{1}$ The balance of angular momentum is not given in its general form; the symmetry of $\mathbf{T}$ guarantees that balance of angular momentum is fulfilled in the absence of body couples.

${ }^{2}$ The Polar Decomposition Theorem states that $\mathbf{F}$ can be decomposed as

$$
\mathbf{F}=\mathbf{R} \mathbf{U}=\mathbf{V R}^{T}
$$

where $\mathbf{R}$ is an orthogonal matrix.
} 
The deformation gradient and the velocity gradient are related through

$$
\frac{\mathrm{d} \mathbf{F}}{\mathrm{d} t}=\mathbf{L F} \text {. }
$$

If the material is incompressible, then $\operatorname{det} \mathbf{F}=1$, which, in turn, implies that $\operatorname{div}_{x} \boldsymbol{v}=\operatorname{tr} \mathbf{L}=\operatorname{tr} \mathbf{D}=$ 0 . In the latter case, incompressibility then leads one to working with the deviatoric (traceless) part of the tensors. If $\mathbf{A}$ is a tensor, then its deviatoric part is denoted by $\mathbf{A}^{d}:=\mathbf{A}-\frac{1}{3}(\operatorname{tr} \mathbf{A}) \mathbf{I}$. In particular, for incompressible fluids and the Cauchy stress $\mathbf{T}$, the quantity $-\frac{1}{3}(\operatorname{tr} \mathbf{T})$ is the mean normal stress, also called the textitmechanical pressure.

The linearized strain is defined through

$$
\varepsilon=\varepsilon(\boldsymbol{u}):=\frac{1}{2}\left(\nabla_{X} \boldsymbol{u}+\left(\nabla_{X} \boldsymbol{u}\right)^{T}\right) .
$$

Since $\mathbf{F}=\nabla_{X} \boldsymbol{u}+\mathbf{I}$, where $\mathbf{I}$ is the identity tensor, one observes that

$$
\mathbf{B}=\mathbf{I}+2 \varepsilon+\left(\nabla_{X} \boldsymbol{u}\right)\left(\nabla_{X} \boldsymbol{u}\right)^{T} .
$$

From linear models in fluid and solid mechanics to materials described by implicit constitutive relations. The classical Hookean linearized approximation to describe the response of elastic solids (see Hooke reprinted in Gunther [27] and the Navier-Stokes fluid model (see Navier $[38,37]$, Poisson [42], Saint-Venant [64], Stokes [68]) are explicit constitutive models in that the stress can be expressed explicitly in terms of the kinematical variable (or for that matter the appropriate kinematical variable in terms of the stress). For example, in the case of incompressible Navier-Stokes fluids, one has

$$
\mathbf{T}=-p \mathbf{I}+2 \mu \mathbf{D} \quad \text { or, equivalently, } \quad \mathbf{D}=\frac{1}{2 \mu} \mathbf{T}^{d},
$$

where $\mu>0$ is the viscosity.

While models with such explicit constitutive relations have been resoundingly successful in describing the response of a variety of solids and fluids, they are quite inadequate when it comes to describing the behavior of many other materials, thereby creating the need for more general constitutive models. Some of these generalizations have led to implicit constitutive theories wherein an implicit relationship is provided between the stress and its properly objective time derivatives and appropriate kinematical quantities and their objective time derivatives. Such models have been used to describe the response of non-Newtonian fluids and inelastic solids.

When attention is restricted to fluids, the model due to Burgers (Burgers [10]) is one such early implicit model (the classical Maxwell model (Maxwell [35]) is not an fully implicit model as one can explicitly describe the symmetric part of the velocity gradient in terms of the stress and its material time derivative). The models developed by Maxwell [35] and Burgers [10] were one-dimensional models; it was Oldroyd [39] who developed properly invariant three-dimensional nonlinear models to describe the response of nonlinear fluids that are truly implicit models, which relate the stress and its appropriate time derivatives to the symmetric part of the velocity gradient and its appropriate time derivatives. While most nonstandard implicit models involve both the stress and its time derivatives as well as the appropriate kinematical variable and its time derivative, there are materials for which one has an implicit relationship between just the stress and an appropriate kinematical quantity; the motivation for the need for such models stems from the existence of viscous fluids whose viscosity depends on the mean normal stress (such fluids are sometimes referred to as piezo-viscous fluids, see Szeri [69]). Similarly, implicit models arise naturally within the context of polymeric fluids with pressure dependent viscosity (see Singh and Nolle [66], McKinney and Belcher [36]), geological materials, which are viscoelastic fluids that have material moduli that depend on the mean normal stress (see the discussion and references in Karra et al [30]), certain biological elastic solids (see the discussion in Freed [22] and Freed and Einstein [23]), and elastomeric solids whose material moduli depend on the mean normal stress (see the discussion in Rajagopal and Saccomandi [56]).

Prǔša and Rajagopal [45] introduced very general implicit constitutive relations of the form

$$
\mathcal{F}_{s=0}^{\infty}[\varrho(t-s), \mathbf{F}(t-s), \mathbf{T}(t-s)]=\mathcal{O},
$$


where $\mathcal{F}_{s=0}^{\infty}$ is a mapping of histories of the density, deformation gradient and stress. They showed that the implicit model (18) contains both rate type models of the Maxwell and Oldroyd-B type as well as differential type models such as the Rivlin-Ericksen fluids (see Rivlin and Ericksen [63]), within the context of approximations based on fading memory (see Coleman and Noll [18]). While the class of implicit models, within the context of special retarded motions, can be approximated by expressions that take the form of the Maxwell, Oldroyd-B and Rivlin-Ericksen fluid models, the approximation procedure does not lead to "models" as the implicit relation (18) does not reduce to these constitutive representations for flows other than those special retarded motions; this point cannot be overemphasized (see Dunn and Rajagopal [21] for a detailed discussion of the relevant issues).

Recently, Rajagopal $[46,47]$ proposed an implicit relationship between the Cauchy stress and the symmetric part of the velocity gradient to describe the response of fluids. Such models, written in the form

$$
\mathbf{G}(\mathbf{T}, \mathbf{D})=\mathbf{0} \quad \text { or } \quad \mathbf{G}\left(\mathbf{T}^{d}, \mathbf{D}\right)=\mathbf{O},
$$

can describe the response of fluids whose viscosity depends on the mean normal stress in the fluid. The representation for such models can also be obtained in special retarded motions of (18). In general, implicit models such as (18) are too general to be of use, as one cannot fashion an experimental program that will lead to measuring the material functions that characterize such models; one has to use much simpler implicit models that are sub-classes of (18).

On turning our attention to the response of solids, we find that implicit models have been developed to describe the inelastic response of a solid, wherein the "yield" condition, which determines the limits of the elastic response, is specified by some measure of the stress. While Maxwell developed the first model to describe the inelastic response of solids, his model, like that of his model for fluids, is not an implicit model. The first implicit model to describe the inelastic response of solids is due to Prandtl [43] and another early model is due to Reuss [61]. Models for describing the elastic response of solids were however explicit models until recently when Rajagopal [46, 47, 48] introduced constitutive models to describe the response of elastic solids, wherein the stress and the deformation gradient are related implicitly. Of particular relevance to our paper are such implicit models that describe the elastic responses of solids.

\section{ElASTIC SOLIDS DESCRIBED BY IMPLICIT CONSTITUTIVE RELATIONS, PARTICULARLY WITH LIMITING SMALL STRAIN}

The focus of the present article is the mathematics and mechanics of a particular sub-class of elastic bodies, which are defined through implicit constitutive relations where the linearized strain is a nonlinear function of the stress and exhibits a limiting strain irrespective of the stress to which the body is subject ${ }^{3}$. Before embarking on a detailed description this class of models, we present a brief review of the development of the theory of elasticity and the need for the new class of models that we shall study.

Although no body is truly "elastic" in that there is no body that does not dissipate energy when deformed (converting mechanical working into energy in thermal form), there are many bodies wherein the dissipation is small enough that it can be ignored whereby making elasticity the most popular theory for describing the response of solids. Even before the celebrated work of Hooke, rudimentary understanding of elasticity was in place (see the discussion in Todhunter [72]). Hooke's law is a one-dimensional constitutive equation that relates the force acting on an elastic body to its extension. This was later generalized by Navier [38, 37] using molecular arguments to obtain a special case of what is currently referred to as the generalized Hooke's law. ${ }^{4}$ Poisson [41, 42] also worked extensively in the field of elasticity and he, too, appealed to molecular

\footnotetext{
${ }^{3}$ The class of models that we consider has a common feature with the classical model of linearized elasticity in that the linearized strain is not Galilean invariant and the class can only be viewed as an approximation of the class of models where the Cauchy-Green tensor $\mathbf{B}$, rather than the linearized strain $\varepsilon$, depends nonlinearly on the stress.

${ }^{4}$ Navier's model was the special case of the constitutive expression (20), where two Lamé constants, $\lambda$ and $\mu$, were taken to be equal.
} 
methods to develop a three-dimensional theory for the response of elastic bodies. Cauchy, who was appointed as one of the commissioners to read Navier's paper on elasticity was probably influenced by the work of Navier with regard to his seminal work on finite elasticity.

In the case of a classical homogeneous isotropic linearized elastic solid one can either express the Cauchy stress in terms of the linearized strain as

$$
\mathbf{T}=\lambda(\operatorname{tr} \boldsymbol{\varepsilon}) \mathbf{I}+2 \mu \boldsymbol{\varepsilon},
$$

where $\lambda$ and $\mu$ are referred to as the Lamé constants, or, equivalently, the linearized strain can be expressed in terms of the Cauchy stress as

$$
\varepsilon=-\frac{\nu}{E}(\operatorname{tr} \mathbf{T}) \mathbf{I}+\frac{(1+\nu)}{E} \mathbf{T}
$$

where $E$ and $\nu$ are the Young's modulus and Poisson's ratio, respectively. The constitutive specification (21) is more in keeping with the demands of causality as the stress is the cause for the deformation of the body. It is worth observing that one cannot perform an experiment in which the Lamé constant $\lambda$ can be determined explicitly (one can measure the Lamé constant $\mu$ and one can measure the bulk modulus $3 \lambda+2 \mu$ ). On the other hand both the Young's modulus $E$ and the Poisson's ratio $\nu$ can be measured directly.

We now turn to discussing compelling reasons for the development of implicit models to describe the response of elastic bodies. Cauchy elasticity is inadequate for the description of a whole host of available experiments concerning the nondissipative response (i.e. response where the dissipation is so small that it can be neglected) of numerous solids. A representative example is the recent experimental work on Gum metal (see Saito et al. [65]) wherein the relationship between the strain and the stress is nonlinear even when the strains are so small (we shall say that a quantity is small if the square of the quantity can be neglected with respect to the quantity). Such a regime for the strain would fall into the regime where classical linearized theory of elasticity would apply as the square of the displacement gradient can be neglected compared to the displacement gradient. All Cauchy elastic models in this strain regime would collapse to the linearized elastic model, but such a model is incapable of describing the nonlinear response that is observed experimentally. The experiments of Saito et al. [65] are not isolated ones: there are numerous other experiments on metallic alloys where one, once again, finds such nonlinear relationships between the strain and the stress when the strains are very small (see the experiments of Li et al. [33], Talling et al. [70], Withey et al. [74], Zhang et al. [75] to mention a few). We immediately recognize the need for a theory of elasticity, more general than Cauchy elasticity, in order to describe such experiments. The models proposed by Rajagopal [55] are capable of accurately describing these experiments (see Figure 6). We now proceed to develop the theory that will lead to such models.

An interesting class of models that are a sub-class of the generalization introduced by Rajagopal is the class of models with a limited stretch. A simple example is a linear spring and an inextensible wire in parallel, where the force cannot be expressed as a function of the elongation (see Figure 3); on the other hand, the elongation can be expressed as a function of the force. Within the context of three dimensional bodies, we recognize that Cauchy elasticity would be incapable of describing the limiting strain response of such elastic bodies. A similar situation presents itself in the case of fluid flow models. A Bingham fluid, which is a fluid with a shear stress threshold ${ }^{5}$ and with regard to such fluids, one cannot express the stress a function of the shear rate. However, one can express the shear rate as a function of the stress.

Another of the failures of the classical linearized theory of elasticity is its inability to explain the strains and stresses at the tip of cracks of brittle elastic bodies. Brittle solid bodies fracture at very small strains without undergoing any inelastic response and such bodies can be described within the ambit of elastic bodies, however the response of such bodies cannot be described adequately by the classical linearized theory, which predicts that the strains can grow like $O\left(r^{-\frac{1}{2}}\right)$, where $r$ is the distance from the crack tip, as $r \rightarrow 0$, a result that contradicts the very assumption under which

\footnotetext{
${ }^{5}$ If by a fluid we mean a body that cannot resist shear stress, then by definition a Bingham material cannot be a fluid. However, it is a commonly accepted practice to refer to the Bingham material as a fluid with a shear stress threshold.
} 


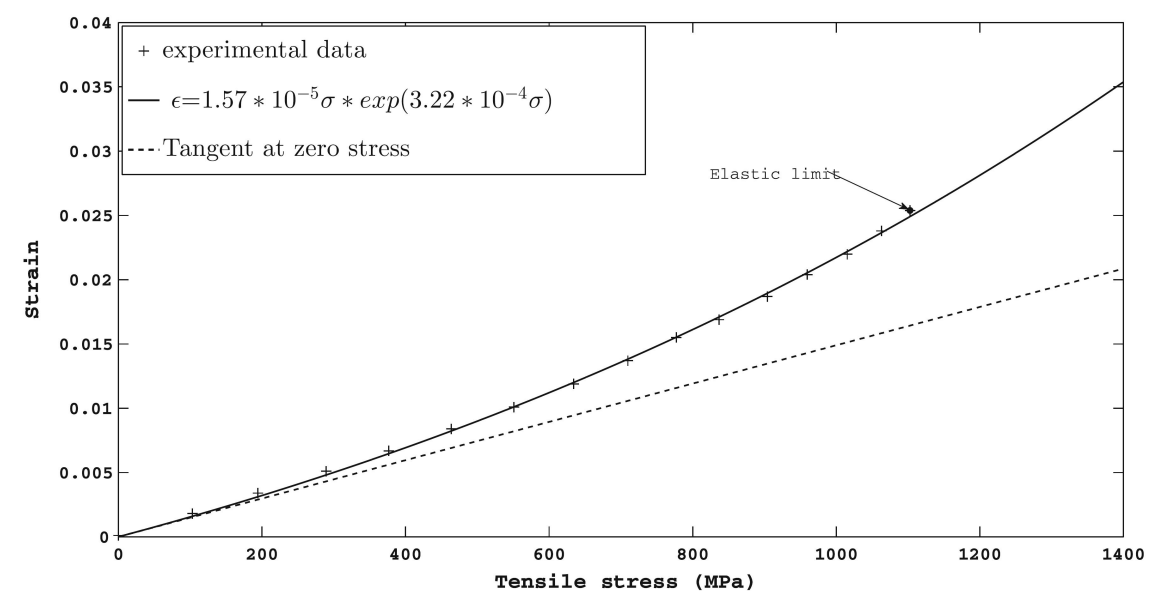

FiguRE 6. Strain-Stress relation for a Gum metal alloy (from Saito et al. [65]). The dotted line shows that the classical linearized response underestimates the strain significantly.

the linearized theory is derived. The study of problems within the context of nonlinear elasticity has not resolved the unbounded growth of the strain thus far (see Knowles and Sternberg [31]). However in the case of a very special class of bodies that meet the Bell constraint ${ }^{6}$ the stresses have been shown to remain bounded (see Tarantino [71]). In any event, one cannot appeal to large deformation theories when the strain is small enough that the linearized theory would apply. On the other hand, the generalization by Rajagopal of the class of elastic bodies affords one the ability to describe bounded strain and stress at crack tips, at tips of notches, etc. (see Rajagopal and Walton [60], Gou et al. [24], Bulíček et al. [8], Kulvait et al. [32]).

We now turn to a brief discussion of the generalization of the class of elastic bodies introduced by Rajagopal $[46,48,51]$. Cauchy and Green elasticity presume that the stress and the stored energy depend on the deformation gradient, respectively ${ }^{7}$. An interesting open question that comes to mind is whether one could have elastic bodies that are more general than Cauchy elastic bodies in that one does not assume that the stress or the stored energy depend on the strain. Rajagopal $[46,48]$ recently showed that the class of elastic bodies is indeed far larger than Cauchy elastic bodies; he considered elastic bodies where one has an implicit relationship between an appropriate stress measure and an appropriate deformation or strain measure of the form:

$$
\mathbf{G}(\mathbf{T}, \mathbf{F})=\mathbf{O} \text {, }
$$

or

$$
\mathbf{H}(\mathbf{S}, \mathbf{E})=\mathbf{O} \text {, }
$$

where the first Piola-Kirchhoff stress $\mathbf{S}$ and the Green-St.Venant strain $\mathbf{E}$ are introduced in (10) and (11), respectively.

Here we shall consider the class of implicit models defined through (22). We shall also restrict our attention to isotropic bodies (see Rajagopal [54] for a discussion of material symmetry issues

\footnotetext{
${ }^{6} \mathrm{~A}$ body that satisfies the Bell constraint is incapable of undergoing simple shear. Also, Bell's experiments concerned the inelastic response of solids and not purely elastic response. To date there have been no experiments that document that the constraint holds within a purely elastic regime.

${ }^{7}$ Green elastic bodies are a sub-class of Cauchy elastic bodies. Cauchy $[16,17]$ did not presume the existence of a stored energy, which depends on the deformation gradient, from which the stress can be derived. This development, which is usually referred to as hyperelasticity or Green elasticity is due to Green [25, 26]. Green [25, 26] recognized that if the stress in an elastic body is not derivable from a potential, which is usually referred to as the stored energy, then one could use such a body as an infinite source of energy. He however did not give a clear proof of this. Rivlin [62] also realized that an elastic body whose stress is not derivable from a potential could give rise to peculiar and unacceptable response characteristics but he did not prove the result either. It was left to Carroll [15] to show that a Cauchy elastic body that is not Green elastic could be used to develop a perpetual motion machine.
} 
for implicit constitutive theories). In this case, we are interested in the class of implicit models defined through

$$
\mathbf{G}(\mathbf{T}, \mathbf{B})=\mathbf{O}
$$

where $\mathbf{B}$ is the left Cauchy-Green tensor, $\mathbf{B}=\mathbf{F F}^{T}$. Since the body is isotropic, it has to satisfy

$$
\mathbf{G}\left(\mathbf{Q} \mathbf{T} \mathbf{Q}^{T}, \mathbf{Q B Q}^{T}\right)=\mathbf{Q} \mathbf{G}(\mathbf{T}, \mathbf{B}) \mathbf{Q}^{T} \quad \forall \mathbf{Q} \in \mathrm{SO}(3),
$$

where $\mathcal{O}$ is the orthogonal group. This leads to the following representation of $\mathbf{G}$ (see Spencer $[67]):$

$$
\begin{aligned}
\mathbf{G}(\mathbf{T}, \mathbf{B}):=\alpha_{0} \mathbf{I} & +\alpha_{1} \mathbf{T}+\alpha_{2} \mathbf{B}+\alpha_{3} \mathbf{T}^{2}+\alpha_{4} \mathbf{B}^{2}+\alpha_{5}(\mathbf{T B}+\mathbf{B} \mathbf{T}) \\
& +\alpha_{6}\left(\mathbf{T}^{2} \mathbf{B}+\mathbf{B} \mathbf{T}^{2}\right)+\alpha_{7}\left(\mathbf{T}^{2} \mathbf{B}^{2}+\mathbf{B}^{2} \mathbf{T}^{2}\right)=\mathbf{0},
\end{aligned}
$$

where the material moduli $\alpha_{i}, i=0, \ldots, 8$, depend upon the density and the invariants

$$
\operatorname{tr} \mathbf{T}, \operatorname{tr} \mathbf{B}, \operatorname{tr} \mathbf{T}^{2}, \operatorname{tr} \mathbf{B}^{2}, \operatorname{tr} \mathbf{T}^{3}, \operatorname{tr} \mathbf{B}^{3}, \operatorname{tr}(\mathbf{T B}), \operatorname{tr}\left(\mathbf{T}^{2} \mathbf{B}\right), \operatorname{tr}\left(\mathbf{T} \mathbf{B}^{2}\right), \operatorname{tr}\left(\mathbf{T}^{2} \mathbf{B}^{2}\right) \text {. }
$$

A special sub-class of the above class of models (26) is given by:

$$
\mathbf{B}=\beta_{0} \mathbf{I}+\beta_{1} \mathbf{T}+\beta_{2} \mathbf{T}^{2},
$$

where the material moduli $\beta_{i}, i=0,1,2$, depend on $\operatorname{tr} \mathbf{T}, \operatorname{tr} \mathbf{T}^{2}, \operatorname{tr} \mathbf{T}^{3}$.

The most general model for a homogeneous, isotropic Cauchy elastic body characterized through the relation

$$
\mathbf{T}=\mathbf{G}(\mathbf{B})
$$

is given by

$$
\mathbf{T}=\gamma_{0} \mathbf{I}+\gamma_{1} \mathbf{B}+\gamma_{2} \mathbf{B}^{2},
$$

where the material moduli $\gamma_{i}, i=0,1,2$, depend on $\operatorname{tr} \mathbf{B}, \operatorname{tr} \mathbf{B}^{2}, \operatorname{tr} \mathbf{B}^{3}$. The class of models (30) cannot in general be obtained from (28) as the expression (28) might not be invertible. Truesdell and Moon [73] investigated when (28) is invertible. However, Truesdell and Moon [73] were not interested in developing models for elasticity that had the structure (28). Nor did they realize the significance and efficacy of such models to describe phenomena that models of the form (30) are incapable of.

We shall highlight that while the classical linearization based on the displacement gradient being small implies that the classical Cauchy elastic model (29) reduces to the linearized elastic model wherein the strain and the stress are related linearly, linearization, under the same conditions, of model (28) leads to a possibly nonlinear relationship between the linearized strain and the stress.

Indeed, on linearizing (29) under the assumption that

$$
\max _{\boldsymbol{X}, t}\left|\nabla_{\boldsymbol{X}} \boldsymbol{u}\right|=\delta, \quad \delta \ll 1,
$$

we arrive, using (16), at

$$
\mathbf{T}=\mathcal{C} \varepsilon
$$

where $\mathcal{C}$ is a fourth-order tensor depending in general on the spatial and the temporal variable, but is independent of $\boldsymbol{\varepsilon}$. Thus, regardless of what nonlinear function $\mathbf{G}$ appears in (29), the linearization (31) will yield a linear model of the type (32) and there is no way of justifying nonlinear elastic models involving a linearized strain if one starts with a Cauchy elastic material (29).

On the other hand, linearizing (28) under the assumption (31), again using (16), leads to

$$
2 \boldsymbol{\varepsilon}=\left(\beta_{0}-1\right) \mathbf{I}+\beta_{1} \mathbf{T}+\beta_{2} \mathbf{T}^{2},
$$

where the material moduli $\beta_{i}, i=0,1,2$, depend on $\operatorname{tr} \mathbf{T}, \operatorname{tr} \mathbf{T}^{2}, \operatorname{tr} \mathbf{T}^{3}$. This relation includes as a special case the model

$$
\boldsymbol{\varepsilon}=\tilde{\beta}_{0}\left(\operatorname{tr} \mathbf{T}, \operatorname{tr}\left(\mathbf{T}^{d}\right)^{2}\right) \mathbf{I}+\tilde{\beta}_{1}\left(\operatorname{tr} \mathbf{T}, \operatorname{tr}\left(\mathbf{T}^{d}\right)^{2}\right) \mathbf{T} .
$$

We notice that in general (34) (and hence (33)) is a nonlinear relationship between the linearized strain and the stress and allows us to describe the experimental work of Saito et al. [65] and others mentioned earlier (see Fig. 6). 
Furthermore, since (34) can be rewritten as the mapping that assigns to $\operatorname{tr} \mathbf{T}$ and $\operatorname{tr} \mathbf{T}^{d}$ its value for $\operatorname{tr} \varepsilon$ and $\varepsilon^{d}$, we conclude that, if this mapping is invertible, then we arrive at models where $\mathbf{T}$ is a nonlinear function of the small strain $\varepsilon$. This seems to be one of the most significant achievements of implicit constitutive theory in solid mechanics.

One could also provide implicit models for elastic bodies that are of the form:

$$
\mathrm{A}_{0}(\mathrm{~S}, \mathrm{E}) \dot{\mathbf{S}}+\mathrm{A}_{1}(\mathrm{~S}, \mathrm{E}) \dot{\mathrm{E}}=\mathbf{O},
$$

where $\mathbf{S}$ and $\mathbf{E}$ are the Piola-Kirchoff stress tensor and the Green-St.Venant strain, respectively, (see (10) and (11)), and the dot denotes the material time derivative (see Rajagopal and Srinivasa [58]), or of the form

$$
[\mathfrak{A}] \mathbf{E}+[\mathfrak{B}] \mathbf{T}^{*}=\mathbf{O},
$$

where $\mathfrak{A}$ and $\mathfrak{B}$ are functions of the state variables and $\mathbf{T}^{*}$ is the rotated stress tensor. The models (35) and (36) are not equivalent to the implicit models (28) and (30) as (35) and (36) might not be integrable and (28) and (30) might not be differentiable.

Thus far, we have restricted our discussion to purely mechanical issues. Implicit theories for elastic bodies can be placed within a thermodynamic framework (see Rajagopal and Srinivasa $[58,59]$ and Bridges and Rajagopal [4]). Of relevance to the class of strain-limiting models for elasticity considered in this paper is the thermodynamical study of Bridges and Rajagopal [4]. They introduce a Gibbs potential that can depend on the Cauchy stress $\mathbf{T}$, the deformation gradient $\mathbf{F}$, the density $\varrho$ and the temperature $\theta$, i.e.,

$$
\Phi=\Phi(\mathbf{T}, \mathbf{F}, \varrho, \theta) .
$$

They suppose that when the stress is $\mathbf{O}$, the Gibbs potential is zero, that the body tends to the stress-free state when the external stimuli are removed, and that the Gibbs potential is a smooth convex function of the stress. Then, defining the rate of dissipation $\xi$ as

$$
\xi=\mathbf{T} \cdot \mathbf{D}-\frac{\mathrm{d} \Psi}{\mathrm{d} t}-\eta \frac{\mathrm{D} \theta}{\mathrm{D} t}-\frac{\boldsymbol{q}}{\theta} \cdot \nabla \theta \geq 0,
$$

where $\Psi$ is the Helmholtz potential (related to the Gibbs potential through a Legendre transform), $\eta$ is the specific entropy and $\mathbf{Q}$ is the heat flux vector, they obtain the implicit rate type equation

$$
\mathcal{A} \stackrel{\oplus}{\mathbf{V}}+\mathcal{B} \stackrel{\oplus}{\mathbf{T}}=\mathbf{0}
$$

by requiring that the second law of thermodynamics is satisfied. In the above equation $\mathcal{A}$ and $\mathcal{B}$ are fourth-order tensor-valued operators and $\square$ above a tensor signifies the time derivative of an appropriate rotation of the tensor: more precisely, for a tensor $\mathbf{A}$,

$$
\stackrel{\square}{\mathbf{A}}:=\frac{\mathrm{d} \mathbf{A}}{\mathrm{d} t}-\Omega \mathbf{A}+\mathbf{A} \Omega \quad \text { with } \quad \Omega:=\frac{\mathrm{d} \mathbf{R}}{\mathrm{d} t} \mathbf{R}^{T},
$$

where $\mathbf{R}$ is the rotation matrix that comes from the Polar Decomposition Theorem applied to $\mathbf{F}$. The rate $\mathbf{A}$ is called the objective Green-McInnis-Naghdi time derivative.

When one assumes that the Gibbs potential depends on the Cauchy stress $\mathbf{T}$, then it follows (see Bridges and Rajagopal [4]) that

$$
\mathbf{E}_{H}=\ln \mathbf{V}=\frac{\partial \Phi}{\partial \mathbf{T}}
$$

where $\mathbf{E}_{H}$ is referred to as the Hencky strain. Assuming that displacement gradients are small, it then follows that

$$
\varepsilon \simeq \mathbf{E}_{H},
$$

where $\varepsilon$ is the linearized strain and hence we have the relation

$$
\varepsilon=\frac{\partial \Phi}{\partial \mathbf{T}} .
$$


When one picks the special form

$$
\Phi(\mathbf{T}):=\frac{\nu}{2} \int_{0}^{\operatorname{tr}\left(\mathbf{T}^{d}\right)^{2}} \frac{\mu}{\left(1+\gamma s^{\frac{\alpha}{2}}\right)^{\frac{1}{\alpha}}} \mathrm{d} s+\frac{\nu}{2} \int_{0}^{|\operatorname{tr} \mathbf{T}|^{2}} \frac{\beta}{\left(1+\beta s^{\frac{\gamma}{2}}\right)^{\frac{1}{\gamma}}} \mathrm{d} s,
$$

then it follows that the linearized strain is given by

$$
\boldsymbol{\varepsilon}=\nu\left[\frac{\mu \mathbf{T}^{d}}{\left(1+\gamma\left(\operatorname{tr}\left(\mathbf{T}^{d}\right)^{2}\right)^{\frac{\alpha}{2}}\right)^{\frac{1}{\alpha}}}+\frac{\beta(\operatorname{tr} \mathbf{T}) \mathbf{I}}{\left(1+\beta|\operatorname{tr} \mathbf{T}|^{\gamma}\right)^{\frac{1}{\gamma}}}\right] .
$$

A body described by the above constitutive relation exhibits strain-limiting behavior. By making different choices of the Gibbs potential $\Phi$, we can obtain a variety of constitutive relations for the linearized strain, and amongst them the various strain-limiting models that are studied in this paper.

Several simple boundary-value problems have been studied within the context of implicit constitutive theories with limiting small strain. Simple problems concerning uniaxial extension, simple shear, torsion, telescopic shear and circumferential shear, as well as several other inhomogeneous deformations have been studied within the context of specific implicit models as well as explicit models wherein the linearized strain or the Cauchy-Green tensor have been expressed as nonlinear functions of the stress (Rajagopal [48, 53, 52, 55], Bustamante [11], Bustamante and Rajagopal [12, 13, 14], Kulvait et al [32], Ortiz et al. [40], Rajagopal and Saravanan [57], Bridges and Rajagopal [4] to mention some of the studies).

A few unsteady problems have been considered within the context of implicit constitutive theory, describing the response of elastic bodies. Recently, several wave propagation problems have been explored within the class of implicit stress power-law elastic bodies; in particular, wave propagation problems, in bodies described by the linearized strain in terms of the stress, have been considered (see Kannan et al. [29] and Kambapalli et al. [28]). The solutions in the case of such models are markedly different from wave propagation in classical elastic bodies in that the stress wave-form changes shape as the wave speed depends on the magnitude of the stress. Usually, the distortion of waves propagating in solids is attributed to dissipation, but with the new class of elastic bodies we see that such distortions of the wave form are possible in a purely elastic solid.

The rigorous mathematical analysis of boundary-value problems that arise in implicitly constituted strain-limiting models has, so far, focused on proving the existence and uniqueness of solutions for any size of data measured in suitable norms in three particular settings: (i) antiplane stress problems resulting in a two-dimensional scalar nonlinear problem (see [8] where the existence of weak solutions in nonconvex domains for values of the model parameter $\alpha$ in the range $\alpha \in(0,2)$, and in convex domains for the range $\alpha \in(0, \infty)$ was established); (ii) the complete multi-dimensional system in the spatially periodic setting in any number of space dimensions (see [9]); (iii) the complete multi-dimensional system in general bounded domains (see Section 4 in this paper). We state these results in the form of theorems in Section 4. The mathematical analysis in Section 4 in the present work is the first one in the literature with focus on BVPs that arise in strain-limiting models in general three-dimensional domains, and include a system of 9 first-order time-independent nonlinear partial differential equations.

\section{PDE ANALYSIS OF BVP PROBLEMS IN GENERAL DOMAINS}

We consider the following problem. Given an open set $\Omega \subset \mathbb{R}^{d}$, two smooth parts of the boundary $\partial \Omega_{D}, \partial \Omega_{N} \subset \partial \Omega$ such that $\partial \Omega_{N} \cap \partial \Omega_{D}=\emptyset$ and $\overline{\partial \Omega_{D} \cup \partial \Omega_{N}}=\partial \Omega$, the given traction $\boldsymbol{g}: \partial \Omega_{N} \rightarrow \mathbb{R}^{d}$ and an external body force $\boldsymbol{f}: \Omega \rightarrow \mathbb{R}^{d}$, we seek a displacement $\boldsymbol{u}: \Omega \rightarrow \mathbb{R}^{d}$ and a Cauchy stress $\mathbf{T}: \Omega \rightarrow \mathbb{R}^{d \times d}$ such that

$$
\begin{aligned}
-\operatorname{div} \mathbf{T} & =\boldsymbol{f} & & \text { in } \Omega, \\
\boldsymbol{\varepsilon}(\boldsymbol{u}) & =\lambda(\operatorname{tr} \mathbf{T})(\operatorname{tr} \mathbf{T}) \mathbf{I}+\mu\left(\left|\mathbf{T}^{d}\right|\right) \mathbf{T}^{d} & & \text { in } \Omega, \\
\boldsymbol{u} & =\mathbf{0} & & \text { on } \partial \Omega_{D}, \\
\mathbf{T} \boldsymbol{n} & =\boldsymbol{g} & & \text { on } \partial \Omega_{N},
\end{aligned}
$$


where $\boldsymbol{\varepsilon}(\boldsymbol{u})$ is the strain tensor associated with the displacement $\boldsymbol{u}$, i.e., $\boldsymbol{\varepsilon}(\boldsymbol{u}):=\frac{1}{2}\left(\nabla \boldsymbol{u}+(\nabla \boldsymbol{u})^{\mathrm{T}}\right)$, $\mathbf{T}^{d}$ denotes the deviatoric (traceless) part of $\mathbf{T}$, i.e., $\mathbf{T}^{d}:=\mathbf{T}-\frac{\operatorname{tr} \mathbf{T}}{d} \mathbf{l}$, and $\boldsymbol{n}$ denotes the unit outward vector at $\partial \Omega$. We shall further assume that the external force is of the form $\boldsymbol{f}:=-\operatorname{div} \mathbf{F}$, where $\mathbf{F}: \Omega \rightarrow \mathbb{R}^{d \times d}$ is a symmetric tensor field.

Assumptions and notation. We begin by introducing suitable structural assumptions on the form of the Cauchy stress. More precisely, we assume that the functions $\lambda$ and $\mu$ appearing in (45) are such that $\lambda \in \mathcal{C}^{1}(\mathbb{R})$ and $\mu \in \mathcal{C}^{1}\left(\mathbb{R}_{+}\right)$; we further require the existence of positive constants $\kappa$, $C_{1}, C_{2}$ and $\alpha$, such that

$$
\begin{aligned}
\frac{C_{1} s^{2}}{\kappa+|s|} & \leq \lambda(s) s^{2} \leq C_{2} s & & \text { for all } s \in \mathbb{R}, \\
\frac{C_{1} s^{2}}{\kappa+s} & \leq \mu(s) s^{2} \leq C_{2} s & & \text { for all } s \in \mathbb{R}_{+}, \\
0 & \leq \frac{\mathrm{d}}{\mathrm{d} s}(\lambda(s) s) & & \text { for all } s \in \mathbb{R}, \\
\frac{C_{1}}{(\kappa+s)^{\alpha+1}} & \leq \frac{\mathrm{d}}{\mathrm{d} s}(\mu(s) s) & & \text { for all } s \in \mathbb{R}_{+} .
\end{aligned}
$$

If (A3) holds with strict inequality, we shall say that $\lambda$ satisfies $\left(\mathrm{A} 3^{\prime}\right)$.

Prototypical examples of $\lambda$ and $\mu$ fulfilling (A1)-(A4) are of the form

$$
\lambda(s):=\frac{1}{\left(1+|s|^{\gamma}\right)^{\frac{1}{\gamma}}}, \quad \mu(s):=\frac{1}{\left(1+s^{\alpha}\right)^{\frac{1}{\alpha}}},
$$

with $\alpha>0$ and $\gamma>0$, see also (44).

Henceforth, $L^{p}(\Omega)$ and $W^{k, p}(\Omega)$ will denote the standard Lebesgue and Sobolev spaces and the corresponding spaces of $d$-component vector-functions and $d \times d$-component tensor-valued functions will be denoted, respectively, by $L^{p}(\Omega)^{d}, L^{p}(\Omega)^{d \times d}$ and $W^{k, p}(\Omega)^{d}$ and $W^{k, p}(\Omega)^{d \times d}$. In order to characterize displacements that vanish on the boundary, $\partial \Omega$, of $\Omega$, we consider the Sobolev space $W_{0}^{1, p}(\Omega)$, defined as the closure of the linear space $\mathcal{D}(\Omega)$, consisting of infinitely many times continuously differentiable functions with compact support in $\Omega$, in the norm of the space $W^{1, p}(\Omega)$ :

$$
W_{0}^{1, p}(\Omega):=\overline{\mathcal{D}(\Omega)}\|\cdot\|_{1, p},
$$

where $p \in[1, \infty)$ is arbitrary. In the same spirit as above, we introduce the vector- and tensorvalued counterparts of $W_{0}^{1, p}(\Omega)$.

In our analysis of the problem we shall also require fractional-order Nikolskiı spaces $\mathcal{N}^{\beta, p}\left(\mathbb{R}^{d}\right)$ for $\beta \in[0,1)$ and $p \in[1, \infty]$, so we shall describe these spaces here briefly. To do so, let us first define

$$
\triangle_{i}^{h} f(x):=f\left(x+h e_{i}\right)-f(x),
$$

where $e_{i}$ is the unit vector pointing in the (positive) $i$-th co-ordinate direction. We then define

$$
\mathcal{N}^{\beta, p}\left(\mathbb{R}^{d}\right):=\left\{f \in L^{p}\left(\mathbb{R}^{d}\right): \sup _{h>0} \sup _{i=1, \ldots, d}\left\|\frac{\triangle_{i}^{h} f}{h^{\beta}}\right\|_{p}<\infty\right\},
$$

equipped with the norm

$$
\|f\|_{\mathcal{N}^{\beta, p}}:=\|f\|_{p}+\sup _{h>0} \sup _{i=1, \ldots, d}\left\|\frac{\triangle_{i}^{h} f}{h^{\beta}}\right\|_{p} .
$$

It is known that $\mathcal{N}^{\beta, p}\left(\mathbb{R}^{d}\right)$ is a Banach space, which is, for $p \in(1, \infty)$, separable and reflexive. The local counterpart of $\mathcal{N}^{\beta, p}\left(\mathbb{R}^{d}\right)$, denoted by $\mathcal{N}_{l o c}^{\beta, p}(\Omega)$, is defined similarly. 
Statement of the main results. Having introduced the necessary notations, we can recall the known results and state the main result of the paper regarding the existence, uniqueness and regularity of the solution to problem (45). The first positive result concerning the existence and uniqueness of a weak solution to the problem (45) under assumptions (A1)-(A4) was established in [8] in a very special geometry, in the case of the so-called anti-plane stress problem. To be more precise, the following theorem was proved in [8].

Theorem 4.1. Let $\Omega_{0} \subset \mathbb{R}^{2}$ be a simply connected Lipschitz set. Define $\Omega:=\Omega_{0} \times \mathbb{R}$ and consider the problem (45) with $\partial \Omega_{D}:=\emptyset$ and $\partial \Omega_{N}:=\partial \Omega_{0} \times \mathbb{R}$. Moreover, assume that $\boldsymbol{f} \equiv \mathbf{0}$ and $\boldsymbol{g} \in \mathcal{C}^{\infty}\left(\partial \Omega_{N} ; \mathbb{R}^{3}\right)$ is of the form $\boldsymbol{g}(\boldsymbol{x})=\left(0,0, g\left(x_{1}, x_{2}\right)\right)$. In addition, let $(\mathrm{A} 1)-(\mathrm{A} 4)$ be satisfied. Then, there exists a unique weak solution to the problem (45), provided that one of the following conditions holds:

(a) $\alpha \in(0, \infty)$ is arbitrary and $\Omega_{0}$ is uniformly convex;

(b) $\alpha \in(0,2)$ and $\partial \Omega_{0}=\Gamma_{\text {con }} \cup \Gamma_{\text {flat }}$, where $\Gamma_{\text {con }}$ is uniformly convex, fulfilling in addition for all $x_{0} \in \Gamma_{\text {con }}$ that $\boldsymbol{\tau} \cap \Omega_{0}=\left\{x_{0}\right\}$, where $\boldsymbol{\tau}$ is any tangent vector to the boundary at the point $x_{0}$, and $\Gamma_{\text {flat }}$ is piece-wise flat, i.e., it is a graph of an affine mapping. Moreover, it is required that $g$ is constant on each flat part of $\partial \Omega_{0}$.

We would like to emphasize here, that Theorem 4.1 is the first positive result for a strain-limiting model. Despite its very special setting, it provides useful insight into the problem. Indeed, it is shown in [8], that in the anti-plane geometry, the problem (45) has a weak solution if and only if the following boundary-value problem has a weak solution:

$$
\begin{aligned}
-\operatorname{div}(\mu(|\nabla U|) \nabla U) & =0 & & \text { in } \Omega_{0}, \\
U & =U_{0} & & \text { on } \partial \Omega_{0},
\end{aligned}
$$

where $U_{0}$ is smooth function given by $\boldsymbol{g}$. Note that the problem (47) falls into the class of elliptic equations with linear growth and as a special case one obtains the minimal surface equation by setting $\alpha:=2$ in (46). It is well known that the solution to (47) does not exists in general and therefore we cannot, in general, guarantee the existence of a solution to (45). On the other hand, it was shown by Bildhauer and Fuchs $[1,2,3]$ that for the convex domains the problem (47) possesses a weak solution, and that for $\alpha \in(0,2)$ and general domains the only problematic behavior of the solution is near the boundary $\partial \Omega_{0}$. Theorem 4.1 however shows that, in some special cases, one can obtain a weak solution in nonconvex domains as well.

The second positive result concerning the problem (45) is contained in [9], where the authors consider the problem with the spatially periodic boundary condition and a less general constitutive equation for $\boldsymbol{\varepsilon}(\boldsymbol{u})$ than that given in (45), namely they considered

$$
\varepsilon(u)=\frac{\mathbf{T}}{\left(1+|\mathbf{T}|^{\alpha}\right)^{\frac{1}{\alpha}}} .
$$

For such a simplified problem they introduced a notion of renormalized solution and established the following result (cf. [9]).

Theorem 4.2. Let $\Omega:=(-1,1)^{d}$ be a cube, $\boldsymbol{f} \in \mathcal{C}^{\infty}\left(\mathbb{R}^{d} ; \mathbb{R}^{d}\right)$ be $\Omega$-periodic and let $\alpha>0$ be arbitrary. Then, there exists a couple $(\boldsymbol{u}, \mathbf{T}) \in W^{1,1}(\Omega)^{d} \times L^{1}(\Omega)^{d \times d}$ with $\boldsymbol{u}$ being $\Omega$-periodic and fulfilling (48), such that

$$
\int_{\Omega} \mathbf{T} \cdot \boldsymbol{\varepsilon}(\boldsymbol{v})+\langle\chi, \boldsymbol{\varepsilon}(\boldsymbol{v})\rangle=\int_{\Omega} \boldsymbol{f} \cdot \boldsymbol{v} \quad \forall \boldsymbol{v} \in \mathcal{C}^{1}\left(\mathbb{R}^{d}\right)^{d} \text { being } \Omega \text {-periodic },
$$

where $\chi \in \mathcal{M}(\Omega)^{d \times d}$ is a symmetric periodic Radon measure that is not absolutely continuous with respect to the Lebesgue measure and is supported on a subset of $\Omega$ of zero Lebesgue measure. Moreover, the following energy inequality holds:

$$
\int_{\Omega} \mathrm{T} \cdot \varepsilon(\boldsymbol{u}) \leq \int_{\Omega} \boldsymbol{f} \cdot \boldsymbol{u} .
$$


In addition, for any $g \in \mathcal{D}(\mathbb{R})$ and any $\boldsymbol{v} \in C^{\infty}\left(\mathbb{R}^{d}\right)^{d}$, being $\Omega$-periodic, the following renormalized equation holds:

$$
\int_{\Omega} g(|\mathbf{T}|) \mathbf{T} \cdot \boldsymbol{\varepsilon}(\boldsymbol{v})+\mathbf{T} \cdot(\nabla g(|\mathbf{T}|) \otimes \boldsymbol{v})=\int_{\Omega} \boldsymbol{f} \cdot g(|\mathbf{T}|) \boldsymbol{v} .
$$

Furthermore, if $\alpha \in\left(0, \frac{2}{d}\right)$ then $\chi \equiv 0$ and $(\mathbf{T}, \boldsymbol{u})$ is a unique weak solution.

This theorem provides and alternative to the notion of a weak solution: that of a renormalized solution. It is based on the observation, that we can a priori control

$$
\int_{\Omega} \frac{|\nabla \mathbf{T}|^{2}}{1+|\mathbf{T}|^{1+\alpha}} \leq C(\boldsymbol{f})
$$

From such an information, one can deduce the compactness of approximating sequences for $\varepsilon(u)$ and can identify their limit in (48). Moreover, this estimate ensures the meaningfulness of the second term in (51), where $\nabla g(|\mathbf{T}|)$ appears. Finally, the estimate (52) provides a uniform bound an approximating sequences for $\mathbf{T}$ in the $L^{1+\varepsilon}$ norm for some $\varepsilon>0$, provided that $\alpha<\frac{2}{d}$, which then implies that the Radon measure appearing in (49) must be identically zero for $\alpha \in\left(0, \frac{2}{d}\right)$.

The final result we present and also prove in this paper is the extension of the result established in [9] to the case of bounded domains, subject to a homogeneous Dirichlet boundary condition.

Theorem 4.3. Let $\Omega \subset \mathbb{R}^{d}$ be a bounded open set, $\partial \Omega_{N}=\emptyset$ and assume that $\lambda$ and $\mu$ satisfy (A1)-(A4) with $0 \leq \alpha<\frac{1}{d}$; then, the following statements hold.

(a) For any $\mathbf{F} \in W^{\beta, 1}(\Omega)^{d \times d}$ with $\beta \in(\alpha d, 1)$, there exists a couple $(\boldsymbol{u}, \mathbf{T})$ such that

$$
\begin{aligned}
\mathbf{T} & \in L^{1}(\Omega)^{d \times d}, \\
\boldsymbol{u} & \in W_{0}^{1, p}(\Omega)^{d}, \quad \text { for all } p \in[1, \infty), \\
\boldsymbol{\varepsilon}(\boldsymbol{u}) & \in L^{\infty}(\Omega)^{d \times d},
\end{aligned}
$$

that is a weak solution to problem (45) in the sense that it satisfies

$$
\int_{\Omega} \mathbf{T} \cdot \boldsymbol{\varepsilon}(\boldsymbol{w}) \mathrm{d} x=\int_{\Omega} \mathbf{F} \cdot \boldsymbol{\varepsilon}(\boldsymbol{w}) \mathrm{d} x \quad \text { for all } \boldsymbol{w} \in \mathcal{D}(\Omega)^{d}
$$

and

$$
\boldsymbol{\varepsilon}(\boldsymbol{u})=\lambda(\operatorname{tr} \mathbf{T})(\operatorname{tr} \mathbf{T}) \mathbf{I}+\mu\left(\left|\mathbf{T}^{d}\right|\right) \mathbf{T}^{d} .
$$

(b) Moreover, if $\Omega$ has continuous boundary ( $\Omega \in \mathcal{C}$ for short), then (53) holds for all $\boldsymbol{w} \in$ $W_{0}^{1,1}(\Omega)^{d}$ such that $\boldsymbol{\varepsilon}(\boldsymbol{w}) \in L^{\infty}(\Omega)^{d \times d}$.

(c) In addition $\boldsymbol{u}$ is unique, and if $\lambda$ satisfies

$$
0<\frac{\mathrm{d}}{\mathrm{d} s}(\lambda(s) s)
$$

then also $\mathbf{T}$ is unique.

(d) Furthermore, if $\mathbf{F}$ belongs to $W^{2,2}(\Omega)^{d \times d}$, then $\mathbf{T} \in W_{l o c}^{1, q}(\Omega)^{d \times d}$ with

$$
q \begin{cases}:=2-\frac{2(d-2)(1+\alpha)}{(d-2)(1+\alpha)+d(1-\alpha)} & \text { for } d \geq 3, \\ \in[1,2) & \text { arbitrary for } d=2 .\end{cases}
$$

Auxiliary results. We complete this section by listing some auxiliary technical tools, which will be required in the rest of the paper. Some of them are proved in the Appendix. The first auxiliary result is a collection of simple algebraic inequalities. 
Lemma 4.1 (See [34] or the Appendix). Let $\lambda$ and $\mu$ satisfy (A1)-(A4); then, there exists a positive constant $C$ such that, for all $\mathbf{W}_{1}, \mathbf{W}_{2} \in \mathbb{R}^{d \times d}$, the following inequalities hold:

$$
\begin{aligned}
& \left(\mu\left(\left|\mathbf{W}_{1}\right|\right) \mathbf{W}_{1}-\mu\left(\left|\mathbf{W}_{2}\right|\right) \mathbf{W}_{2}\right) \cdot\left(\mathbf{W}_{1}-\mathbf{W}_{2}\right) \geq \frac{C\left|\mathbf{W}_{1}-\mathbf{W}_{2}\right|^{2}}{\left(\kappa+\left|\mathbf{W}_{1}\right|+\left|\mathbf{W}_{2}\right|\right)^{1+\alpha}} ; \\
& \left(\mu\left(\left|\mathbf{W}_{1}\right|\right) \mathbf{W}_{1}-\mu\left(\left|\mathbf{W}_{2}\right|\right) \mathbf{W}_{2}\right) \cdot\left(\mathbf{W}_{1}-\mathbf{W}_{2}\right) \geq\left|\left(\kappa+\left|\mathbf{W}_{1}\right|\right)^{\frac{1-\alpha}{2}}-\left(\kappa+\left|\mathbf{W}_{2}\right|\right)^{\frac{1-\alpha}{2}}\right|^{2} ; \\
& \left(\lambda\left(\operatorname{tr} \mathbf{W}_{1}\right) \operatorname{tr} \mathbf{W}_{1}-\lambda\left(\operatorname{tr} \mathbf{W}_{2}\right) \operatorname{tr} \mathbf{W}_{2}\right)\left(\operatorname{tr} \mathbf{W}_{1}-\operatorname{tr} \mathbf{W}_{2}\right) \geq 0 .
\end{aligned}
$$

If, in addition, $\lambda$ satisfies (A3'), then the following inequality holds for all $\mathbf{W}_{1}, \mathbf{W}_{2}$ such that $\operatorname{tr} \mathbf{W}_{1} \neq \operatorname{tr} \mathbf{W}_{2}$ :

$$
\left(\lambda\left(\operatorname{tr} \mathbf{W}_{1}\right) \operatorname{tr} \mathbf{W}_{1}-\lambda\left(\operatorname{tr} \mathbf{W}_{2}\right) \operatorname{tr} \mathbf{W}_{2}\right)\left(\operatorname{tr} \mathbf{W}_{1}-\operatorname{tr} \mathbf{W}_{2}\right)>0 .
$$

The second auxiliary result provides a bound on the $L^{p}$ norm of a matrix-valued function in terms of its deviatoric part; its proof is contained in the Appendix.

Lemma 4.2. Let $\Omega \subset \mathbb{R}^{d}$ be a Lipschitz domain $\left(\Omega \in \mathcal{C}^{0,1}\right.$ in short); then, for any $p \in(1, \infty)$, there exists a positive constant $C=C(p, \Omega)$ such that the following inequality holds for all $\mathbf{T} \in L^{p}(\Omega)^{d \times d}$ :

$$
\|\mathbf{T}\|_{p} \leq C\left(\left|\int_{\Omega} \operatorname{tr} \mathbf{T} \mathrm{d} x\right|+\left\|\mathbf{T}^{d}\right\|_{p}+\sup _{\boldsymbol{w} \in W_{0}^{1, p^{\prime}}(\Omega)^{d} ;\|\boldsymbol{w}\|_{1, p^{\prime}} \leq 1} \int_{\Omega} \mathbf{T} \cdot \nabla \boldsymbol{w} \mathrm{d} x\right) .
$$

Moreover, if $\mathbf{T} \in W_{l o c}^{1,1}(\Omega)^{d \times d}$, then

$$
|\nabla \mathbf{T}| \leq C\left(\left|\nabla \mathbf{T}^{d}\right|+|\operatorname{div} \mathbf{T}|\right) \quad \text { a.e. in } \Omega .
$$

Our third auxiliary result is concerned with the weak ${ }^{*}$ density of smooth functions, which is again proved in the Appendix.

Lemma 4.3. Let $\Omega \subset \mathbb{R}^{d}$ be a domain with continuous boundary. Then, for any $\boldsymbol{u} \in W_{0}^{1,1}(\Omega)^{d}$ with $\boldsymbol{\varepsilon}(\boldsymbol{u}) \in L^{\infty}(\Omega)^{d \times d}$, there exists a sequence $\left\{\boldsymbol{u}^{n}\right\}_{n=1}^{\infty}$ such that $\boldsymbol{u}^{n} \in \mathcal{D}(\Omega)^{d}$ for all $n \in \mathbb{N}$ and

$$
\begin{array}{clrl}
\boldsymbol{u}^{n} \rightarrow \boldsymbol{u} & & \text { strongly in } W_{0}^{1, p}(\Omega)^{d} \text { for all } 1 \leq p<\infty, \\
\boldsymbol{\varepsilon}\left(\boldsymbol{u}^{n}\right) \boldsymbol{~}^{*} \boldsymbol{\varepsilon}(\boldsymbol{u}) & & \text { weakly }{ }^{*} \text { in } L^{\infty}(\Omega)^{d \times d} .
\end{array}
$$

Finally, we recall a classical embedding theorem for Nikolskiı spaces.

Lemma 4.4. Let $\beta \in[0,1)$ and $p \in[1, \infty]$ be such that $\beta p<d$; then, there exists a positive constant $C$ such that, for all $f \in \mathcal{N}^{\beta, p}\left(\mathbb{R}^{d}\right)$ and for all small $\delta>0$,

$$
\|f\|_{L^{\frac{d p}{d-\beta p}-\delta}\left(\mathbb{R}^{d}\right)} \leq C\|f\|_{\mathcal{N}^{\beta, p}\left(\mathbb{R}^{d}\right)} .
$$

The rest of the paper is organized as follows. In Section 4.1, we prove the uniqueness part of Theorem 4.3. Next, in Section 4.2 we introduce an approximation scheme, similar to the $p$-Laplace equation, and let $p \rightarrow 1$ to obtain the existence part of Theorem 4.3. Section 4.3 is devoted to the proof of the estimates for the derivatives of $\mathbf{T}$, which is based on a different approximation scheme than the one in Section 4.2. Finally, in the Appendix, we prove some of the auxiliary results that were stated above.

4.1. Uniqueness. In this section we shall assume that the statement (a) of Theorem 4.3 holds and we shall prove that (b) and (c) hold. We shall then prove in Section 4.2 and Section 4.3, respectively, that (a) and (d) hold.

We begin by observing that if (53) and $\Omega \in \mathcal{C}$, then

$$
\int_{\Omega} \mathbf{T} \cdot \boldsymbol{\varepsilon}(\boldsymbol{w}) \mathrm{d} x=\int_{\Omega} \mathbf{F} \cdot \boldsymbol{\varepsilon}(\boldsymbol{w}) \mathrm{d} x
$$

for all $\boldsymbol{w} \in W_{0}^{1,1}(\Omega)$ such that $\boldsymbol{\varepsilon}(\boldsymbol{w}) \in L^{\infty}(\Omega)^{d \times d}$. Indeed, the identity (64) follows from (53) and from the weak* approximation Lemma 4.3. Thus, let $\left(\boldsymbol{u}_{1}, \mathbf{T}_{1}\right)$ and $\left(\boldsymbol{u}_{2}, \mathbf{T}_{2}\right)$ be two weak solutions 
to (45), and define $\boldsymbol{z}:=\boldsymbol{u}_{1}-\boldsymbol{u}_{2}$. Then, upon subtracting (64) for $\left(\boldsymbol{u}_{2}, \mathbf{T}_{2}\right)$ from (64) for $\left(\boldsymbol{u}_{1}, \mathbf{T}_{1}\right)$, we obtain

$$
\int_{\Omega}\left(\mathbf{T}_{1}-\mathbf{T}_{2}\right) \cdot \varepsilon(\boldsymbol{w}) \mathrm{d} x=0
$$

which after using the definition of $\boldsymbol{\varepsilon}(\boldsymbol{z})$ implies that

$$
\begin{aligned}
& \int_{\Omega}(\left.\lambda\left(\operatorname{tr} \mathbf{T}_{1}\right)\left(\operatorname{tr} \mathbf{T}_{1}\right)-\lambda\left(\operatorname{tr} \mathbf{T}_{1}\right)\left(\operatorname{tr} \mathbf{T}_{1}\right)\right) \operatorname{tr}\left(\mathbf{T}_{1}-\mathbf{T}_{2}\right) \mathrm{d} x \\
& \quad+\int_{\Omega}\left(\mu\left(\left|\mathbf{T}_{1}^{d}\right|\right) \mathbf{T}_{1}^{d}-\mu\left(\left|\mathbf{T}_{2}^{d}\right|\right) \mathbf{T}_{2}^{d}\right) \cdot\left(\mathbf{T}_{1}^{d}-\mathbf{T}_{2}^{d}\right) \mathrm{d} x=0 .
\end{aligned}
$$

Hence, using the non-negativity of the integrands (which follows from Lemma 4.1), we get that

$$
\begin{aligned}
\left(\mu\left(\left|\mathbf{T}_{1}^{d}\right|\right) \mathbf{T}_{1}^{d}-\mu\left(\left|\mathbf{T}_{2}^{d}\right|\right) \mathbf{T}_{2}^{d}\right) \cdot\left(\mathbf{T}_{1}^{d}-\mathbf{T}_{2}^{d}\right) & =0, \\
\left(\lambda\left(\operatorname{tr} \mathbf{T}_{1}\right)\left(\operatorname{tr} \mathbf{T}_{1}\right)-\lambda\left(\operatorname{tr} \mathbf{T}_{1}\right)\left(\operatorname{tr} \mathbf{T}_{1}\right)\right)\left(\operatorname{tr} \mathbf{T}_{1}-\operatorname{tr} \mathbf{T}_{2}\right) & =0,
\end{aligned}
$$

almost everywhere in $\Omega$. Thus, it directly follows from (55) that $\mathbf{T}_{1}^{d}=\mathbf{T}_{2}^{d}$ almost everywhere in $\Omega$. Consequently, using the definition of $\boldsymbol{\varepsilon}(\boldsymbol{z})$, we have that

$$
\boldsymbol{\varepsilon}(\boldsymbol{z})-\frac{\operatorname{div} \boldsymbol{z}}{d} \mathbf{I}=\boldsymbol{\varepsilon}(\boldsymbol{z})-\frac{1}{d}(\operatorname{tr} \boldsymbol{\varepsilon}(\boldsymbol{z})) \mathbf{I}=\mu\left(\left|\mathbf{T}_{1}^{d}\right|\right) \mathbf{T}_{1}^{d}-\mu\left(\left|\mathbf{T}_{2}^{d}\right|\right) \mathbf{T}_{2}^{d}=0 .
$$

Therefore, after integration by parts (which can be justified by the density of compactly supported smooth functions according to the definition of $W_{0}^{1,2}(\Omega)$ ), we have that

$$
\begin{aligned}
0 & =\int_{\Omega}\left|\varepsilon(\boldsymbol{z})-\frac{\operatorname{div} \boldsymbol{z}}{d} \mathbf{I}\right|^{2} \mathrm{~d} x=\int_{\Omega}|\varepsilon(\boldsymbol{z})|^{2}-\frac{1}{d}|\operatorname{div} \boldsymbol{z}|^{2} \mathrm{~d} x \\
& =\int_{\Omega} \varepsilon(\boldsymbol{z}) \cdot \nabla \boldsymbol{z}-\frac{1}{d}|\operatorname{div} \boldsymbol{z}|^{2} \mathrm{~d} x=\frac{1}{2} \int_{\Omega}-\triangle \boldsymbol{z} \cdot \boldsymbol{z}-\nabla(\operatorname{div} \boldsymbol{z}) \cdot \boldsymbol{z}-\frac{2}{d}|\operatorname{div} \boldsymbol{z}|^{2} \mathrm{~d} x \\
& =\frac{1}{2} \int_{\Omega}|\nabla \boldsymbol{z}|^{2}+\frac{d-2}{d}|\operatorname{div} \boldsymbol{z}|^{2} \mathrm{~d} x .
\end{aligned}
$$

We thus deduce that $\boldsymbol{u}_{1}=\boldsymbol{u}_{2}$ almost everywhere in $\Omega$. Moreover, if (A3') is valid, it follows from (58) and (66) that also $\operatorname{tr} \mathbf{T}_{1}=\operatorname{tr} \mathbf{T}_{2}$ and consequently, from the above computation we obtain that $\mathbf{T}_{1}=\mathbf{T}_{2}$.

4.2. Existence. This section is devoted to the proof of part (a) of Theorem 4.3. First, we introduce an approximating problem, to which the theory of monotone nonlinear operators can be applied. We then derive the first a priori estimates for $\mathbf{T}$ and $\varepsilon(u)$, which lead to the uniform (independent of the order of approximation) bound $\|\mathbf{T}\|_{1} \leq C$. Next, we improve the integrability of $\mathbf{T}$ to be able to show weak convergence of the sequence of approximations $\mathbf{T}$ in $L^{1}(\Omega)^{d \times d}$, and finally we apply generalized monotone operator theory and show the convergence of the sequence of approximating solutions to the desired weak solution of (45).

Approximating problem. We introduce the following approximating problem: for a positive integer $n$, we seek $(\boldsymbol{u}, \mathbf{T}) \in W_{0}^{1, n+1}(\Omega)^{d} \times L^{1+\frac{1}{n}}(\Omega)^{d \times d}$ such that

$$
\begin{array}{rlrl}
0 & =\int_{\Omega}(\mathbf{F}-\mathbf{T}) \cdot \nabla \boldsymbol{w} \mathrm{d} x \quad \text { for all } \boldsymbol{w} \in W_{0}^{1, n+1}(\Omega)^{d}, \\
\varepsilon(\boldsymbol{u}) & =\lambda(\operatorname{tr} \mathbf{T})(\operatorname{tr} \mathbf{T}) \mathbf{I}+\mu\left(\left|\mathbf{T}^{d}\right|\right) \mathbf{T}^{d}+\frac{\mathbf{T}^{d}}{n\left|\mathbf{T}^{d}\right|^{1-\frac{1}{n}}}+\frac{(\operatorname{tr} \mathbf{T}) \mathbf{I}}{n|\operatorname{tr} \mathbf{T}|^{1-\frac{1}{n}}} \quad \text { in } \Omega, \\
\boldsymbol{u} & =\mathbf{0} \quad \text { on } \partial \Omega . &
\end{array}
$$

First, to ensure the meaningfulness of the term appearing on the right-hand side of (67), we restrict ourselves to $n \geq n_{0}$, where $n_{0}$ is the smallest positive integer such that

$$
W^{\beta, 1}(\Omega) \hookrightarrow L^{1+\frac{1}{n_{0}}}(\Omega) .
$$

It then follows from our assumption on $\mathbf{F}$, that $\mathbf{F} \in L^{1+\frac{1}{n}}(\Omega)^{d \times d}$ for all $n \geq n_{0}$, and thus the equation (67) is meaningful (thanks to Hölder's inequality). In order to show the existence of a 
solution to (67)-(69), it is not difficult to see (using also the monotonicity Lemma 4.1), that (68) can be equivalently rewritten as

$$
\mathbf{T}=\mathbf{T}^{*}(\varepsilon(\boldsymbol{u})),
$$

where $\mathbf{T}^{*}: \mathbb{R}_{\text {sym }}^{d \times d} \rightarrow \mathbb{R}_{\text {sym }}^{d \times d}$ is a continuous mapping fulfilling

$$
\begin{aligned}
& \mathbf{T}^{*}(\mathbf{D}) \cdot \mathbf{D} \geq-C(n)+\tilde{C}(n)|\mathbf{D}|^{n+1}, \quad\left|\mathbf{T}^{*}(\mathbf{D})\right| \leq C(n)\left(1+|\mathbf{D}|^{n}\right), \\
& \left(\mathbf{T}^{*}\left(\mathbf{D}_{1}\right)-\mathbf{T}^{*}\left(\mathbf{D}_{2}\right)\right) \cdot\left(\mathbf{D}_{1}-\mathbf{D}_{2}\right) \geq 0 .
\end{aligned}
$$

Therefore, the solvability of (67)-(69) follows from standard monotone operator theory.

First a priori estimates. Here, we state the relevant a priori estimates. By setting $\boldsymbol{w}:=\boldsymbol{u}$ in (67), we get the identity

$$
\int_{\Omega} \mathbf{T} \cdot \boldsymbol{\varepsilon}(\boldsymbol{u}) \mathrm{d} x=\int_{\Omega} \mathbf{F} \cdot \boldsymbol{\varepsilon}(\boldsymbol{u}) \mathrm{d} x .
$$

Next, using (68) and (A1)-(A2) we obtain

$$
\int_{\Omega} \frac{C_{1}(\operatorname{tr} \mathbf{T})^{2}}{(\kappa+|\operatorname{tr} \mathbf{T}|)}+\frac{C_{1}\left|\mathbf{T}^{d}\right|^{2}}{\left(\kappa+\left|\mathbf{T}^{d}\right|\right)}+\frac{\left|\mathbf{T}^{d}\right|^{1+\frac{1}{n}}}{n}+\frac{|\operatorname{tr} \mathbf{T}|^{1+\frac{1}{n}}}{n} \mathrm{~d} x \leq \int_{\Omega} \mathbf{F} \cdot \boldsymbol{\varepsilon}(\boldsymbol{u}) \mathrm{d} x
$$

Moreover, using (A1)-(A2) in (68), we deduce the following estimate:

$$
|\varepsilon(\boldsymbol{u})| \leq C+\frac{|\mathbf{T}|^{\frac{1}{n}}}{n} .
$$

Substituting (72) into (71), we obtain

$$
\begin{aligned}
& \int_{\Omega} \frac{C_{1}(\operatorname{tr} \mathbf{T})^{2}}{(\kappa+|\operatorname{tr} \mathbf{T}|)}+\frac{C_{1}\left|\mathbf{T}^{d}\right|^{2}}{\left(\kappa+\left|\mathbf{T}^{d}\right|\right)}+\frac{\left|\mathbf{T}^{d}\right|^{1+\frac{1}{n}}}{n}+\frac{|\operatorname{tr} \mathbf{T}|^{1+\frac{1}{n}}}{n} \mathrm{~d} x \\
& \quad \leq \int_{\Omega}|\mathbf{F}|\left(C+\frac{|\mathbf{T}|^{\frac{1}{n}}}{n}\right) \mathrm{d} x \leq C\left(\|\mathbf{F}\|_{1}+\|\mathbf{F}\|_{\frac{n+1}{n}}^{\frac{n+1}{n+1}}\right)+\frac{\|\mathbf{T}\|_{1+\frac{1}{n}}^{1+\frac{1}{n}}}{n^{n+1}},
\end{aligned}
$$

where for the second inequality we used Young's inequality. Consequently, by absorbing the last term into the left-hand side, we get, after a simple algebraic manipulation (note that at this point we restrict ourselves to the case when $n \geq n_{0}$ ), that

$$
\|\mathbf{T}\|_{1}+\frac{\|\mathbf{T}\|_{1+\frac{1}{n}}^{1+\frac{1}{n}}}{n} \leq C,
$$

where $C$ is a positive constant, independent of $n$. Thus, returning to (72), we have that

$$
\|\varepsilon(\boldsymbol{u})\|_{n+1} \leq C,
$$

where, again, $C$ is a positive constant, independent of $n$.

Fractional derivative estimates. The next step is to improve (uniformly with respect to $n$ ) the information about $\mathbf{T}$. To do so, we derive fractional derivative estimates for $\mathbf{T}$, which will finally lead to such an improvement. Hence, we fix an arbitrary $h_{0}>0$, and for any $h \leq h_{0}$ and any $i=1, \ldots, d$, we deduce from (67) that (we recall here that the operator $\triangle_{i}^{h}$ is defined by $\left.\triangle_{i}^{h} f(x):=f\left(x+h e_{i}\right)-f(x)\right)$

$$
\int_{\Omega} \triangle_{i}^{h} \mathbf{T} \cdot \boldsymbol{\varepsilon}(\boldsymbol{w}) \mathrm{d} x=\int_{\Omega} \triangle_{i}^{h} \mathbf{F} \cdot \boldsymbol{\varepsilon}(\boldsymbol{w}) \mathrm{d} x
$$

which holds for any $\boldsymbol{w} \in W^{1, n+1}(\Omega)^{d}$ such that $\boldsymbol{w}(x)=0$ for all $x \in \Omega$ fulfilling $\operatorname{dist}(x, \partial \Omega) \leq h_{0}$. Next, we choose a nonnegative function $\tau \in \mathcal{D}(\Omega)$ such that

$$
\tau(x)= \begin{cases}0 & \operatorname{dist}(x, \partial \Omega) \leq h_{0}, \\ 1 & \operatorname{dist}(x, \partial \Omega) \geq 2 h_{0},\end{cases}
$$

and set

$$
\boldsymbol{w}(x):=\tau \triangle_{i}^{h} \boldsymbol{u}
$$


in (76) to get

$$
\begin{aligned}
\int_{\Omega} \tau \triangle_{i}^{h} \mathbf{T} \cdot \triangle_{i}^{h} \varepsilon(\boldsymbol{u}) \mathrm{d} x= & \int_{\Omega} \tau \triangle_{i}^{h} \mathbf{F} \cdot \triangle_{i}^{h} \varepsilon(\boldsymbol{u}) \mathrm{d} x \\
& +\int_{\Omega}\left(\triangle_{i}^{h} \mathbf{F}-\triangle_{i}^{h} \mathbf{T}\right) \cdot\left(\triangle_{i}^{h} \boldsymbol{u} \otimes \nabla \tau\right) \mathrm{d} x .
\end{aligned}
$$

The left-hand side can be estimated from below by using the monotonicity of the formula (68) (see also the proof of Theorem 4.1) to obtain

$$
\int_{\Omega} \tau \triangle_{i}^{h}\left(\mu\left(\left|\mathbf{T}^{d}\right|\right) \mathbf{T}^{d}\right) \cdot \triangle_{i}^{h} \mathbf{T}^{d} \mathrm{~d} x \leq \int_{\Omega} \tau \triangle_{i}^{h} \mathbf{T} \cdot \triangle_{i}^{h} \boldsymbol{\varepsilon}(\boldsymbol{u}) \mathrm{d} x,
$$

which by using (56) leads to

$$
\int_{\Omega}\left|\triangle_{i}^{h}\left(\left(\kappa+\left|\mathbf{T}^{d}\right|\right)^{\frac{1-\alpha}{2}}\right)\right|^{2} \tau \mathrm{d} x \leq C \int_{\Omega} \tau \triangle_{i}^{h} \mathbf{T} \cdot \triangle_{i}^{h} \boldsymbol{\varepsilon}(\boldsymbol{u}) \mathrm{d} x .
$$

For the first term on the right-hand side of (77), we use Hölder's inequality and the uniform estimate (75) to get

$$
\int_{\Omega} \tau \triangle_{i}^{h} \mathbf{F} \cdot \triangle_{i}^{h} \varepsilon(\boldsymbol{u}) \mathrm{d} x \leq C\left(\int_{\Omega}\left|\triangle_{i}^{h} \mathbf{F}\right|^{\frac{n+1}{n}} \tau \mathrm{d} x\right)^{\frac{n}{n+1}} .
$$

Finally, the last term in (77) is bounded by using Hölder's inequality and the estimate (74) as follows:

$$
\begin{aligned}
\int_{\Omega}\left(\triangle_{i}^{h} \mathbf{F}-\triangle_{i}^{h} \mathbf{T}\right) \cdot\left(\triangle_{i}^{h} \boldsymbol{u} \otimes \nabla \tau\right) \mathrm{d} x & \leq C\left(\|\mathbf{F}\|_{1}+\|\mathbf{T}\|_{1}\right)\left\||\nabla \tau| \triangle_{i}^{h} \boldsymbol{u}\right\|_{\infty} \\
& \leq C\left\||\nabla \tau| \triangle_{i}^{h} \boldsymbol{u}\right\|_{\infty} .
\end{aligned}
$$

Thus, defining

$$
\beta^{n}:=\beta-\frac{d}{n+1}
$$

and combining (78)-(80), we get the inequality

$$
\int_{\Omega}\left|\frac{\triangle_{i}^{h}\left(\kappa+\left|\mathbf{T}^{d}\right|\right)^{\frac{1-\alpha}{2}}}{h^{\frac{\beta^{n}}{2}}}\right|^{2} \tau \mathrm{d} x \leq C\left(\int_{\Omega} \frac{\left|\triangle_{i}^{h} \mathbf{F}\right|^{\frac{n+1}{n}}}{h^{\frac{(n+1) \beta^{n}}{n}}} \tau \mathrm{d} x\right)^{\frac{n}{n+1}}+C \frac{\left\||\nabla \tau| \triangle_{i}^{h} \boldsymbol{u}\right\|_{\infty}}{h^{\beta^{n}}} .
$$

We focus on the uniform bound on the terms on the right-hand side. First, using the embedding theorem (with the embedding constant independent of $n$ ) we find that

$$
W^{\beta, 1}\left(\Omega_{0}\right) \hookrightarrow W^{\beta^{n}, \frac{n+1}{n}}\left(\Omega_{0}\right)
$$

for any smooth $\Omega_{0} \subset \bar{\Omega}_{0} \subset \Omega$ and therefore using the assumption on $\mathbf{F}$ we see that

$$
\int_{\Omega} \frac{\left|\triangle_{i}^{h} \mathbf{F}\right|^{\frac{n+1}{n}}}{h^{\frac{(n+1) \beta^{n}}{n}}} \tau \mathrm{d} x \leq C\left(h_{0}\right) .
$$

To bound also the second term in (82), we need to restrict ourselves to large values of $n$. To be more precise, we find the smallest $n_{1} \in \mathbb{N}$ such that for all $n>n_{1}$ we have

$$
\beta^{n}>0 \text {. }
$$

Then, for such $n$ 's, since $0<\beta^{n}<1$, using Morrey's embedding theorem we find that

$$
W^{1, \frac{d}{1-\beta^{n}}}\left(\Omega_{0}\right) \hookrightarrow \mathcal{C}^{0, \beta^{n}}\left(\Omega_{0}\right) .
$$

In addition, since

$$
d \leq \frac{d}{1-\beta^{n}} \leq \frac{d}{1-\beta}<\infty,
$$

we can use Korn's inequality to deduce the existence of a positive constant $C$, independent of $n$, such that

$$
\|\boldsymbol{u}\|_{\mathcal{C}^{0, \beta^{n}}\left(\Omega_{0}\right)^{d}} \leq C\|\varepsilon(\boldsymbol{u})\|_{\frac{d}{1-\beta^{n}}} .
$$


Next, using the definition of $\beta^{n}$ we observe that

$$
\frac{d}{1-\beta^{n}}=\frac{d}{1-\beta+\frac{d}{n+1}}=\frac{d(n+1)}{(n+1)(1-\beta)+d} \leq n+1 .
$$

Using Hölder's inequality, the fact that $\Omega$ is bounded and (83), we thus deduce that

$$
\begin{aligned}
\frac{\left\|\triangle_{i}^{h} \boldsymbol{u}\right\| \nabla \tau \mid \|_{\infty}}{h^{\beta^{n}}} & \leq C\left(h_{0}\right)\|\boldsymbol{u}\|_{\mathcal{C}^{0, \beta^{n}}\left(\Omega_{0}\right)^{d}} \leq C\left(h_{0}\right)\|\boldsymbol{\varepsilon}(\boldsymbol{u})\|_{\frac{d}{1-\beta^{n}}} \\
& \leq C\left(h_{0}\right)\|\boldsymbol{\varepsilon}(\boldsymbol{u})\|_{n+1} \leq C\left(h_{0}\right),
\end{aligned}
$$

where for the last inequality we used (75). Consequently, substituting these estimates into (82) we get that, for all $n \geq n_{1}$ and all $\Omega_{0} \subset \bar{\Omega}_{0} \subset \Omega$,

$$
\int_{\Omega}\left|\frac{\triangle_{i}^{h}\left(\kappa+\left|\mathbf{T}^{d}\right|\right)^{\frac{1-\alpha}{2}}}{h^{\frac{\beta^{n}}{2}}}\right|^{2} \tau \mathrm{d} x \leq C\left(\Omega_{0}\right)
$$

In addition using the a priori bound (74), we see that (84) leads to

$$
\left\|\left(\kappa+\left|\mathbf{T}^{d}\right|\right)^{\frac{1-\alpha}{2}}\right\|_{\mathcal{N}^{\frac{\beta^{n}}{2}, 2}\left(\Omega_{0}\right)} \leq C\left(\Omega_{0}\right)
$$

for any $\Omega_{0} \subset \bar{\Omega}_{0} \subset \Omega$. Hence, using the embedding theorem, we find that

$$
\left\|\left(\kappa+\left|\mathbf{T}^{d}\right|\right)^{\frac{1-\alpha}{2}}\right\|_{L^{\frac{2 d}{d-\beta^{n}}-\delta}\left(\Omega_{0}\right)} \leq C\left(\Omega_{0}\right) \Longrightarrow\left\|\mathbf{T}^{d}\right\|_{L^{\frac{d(1-\alpha)}{d-\beta+\frac{d}{n+1}}}-\delta} \leq C\left(\Omega_{0}\right) .
$$

Next, since $\beta>\alpha d$ and $\alpha \in(0,1 / d)$, it is meaningful to define

$$
0<p:=\frac{d(1-\alpha)}{d-\beta}-1=\frac{\beta-\alpha d}{d-\beta},
$$

and we can find the minimal $n_{2} \in \mathbb{N}$ such that for all $n \geq n_{2}$ we have

$$
\frac{d(1-\alpha)}{d-\beta+\frac{d}{n+1}}>1+\frac{p}{2} \text {. }
$$

It then follows from (86) that for all $n \geq \max \left(n_{1}, n_{2}\right)$ and all $\Omega_{0} \subset \bar{\Omega}_{0} \subset \Omega$ we have

$$
\left\|\mathbf{T}^{d}\right\|_{L^{1+\frac{p}{2}}\left(\Omega_{0}\right)} \leq C\left(\Omega_{0}\right)
$$

Hence, using (59), the identity (67) (to control div T) and the a priori bound (74) (to control the mean value of $\operatorname{tr} \mathbf{T}$ ), we deduce from (87) the final estimate (valid for all $n \geq \max \left(n_{1}, n_{2}\right)$ ):

$$
\|\mathbf{T}\|_{L^{1+\frac{p}{2}}\left(\Omega_{0}\right)} \leq C\left(\Omega_{0}\right)\|\mathbf{T}\|{\frac{d(1-\alpha)}{L^{d-\beta+\frac{d}{n+1}}}\left(\Omega_{0}\right)} \leq C\left(\Omega_{0}\right) .
$$

Limit $n \rightarrow \infty$. We denote by $\left(\boldsymbol{u}^{n}, \mathbf{T}^{n}\right)$ a solution constructed in the previous section, and assume henceforth that $n \geq \max \left(n_{0}, n_{1}, n_{2}\right)$. It then follows from (74)-(75) and compact Sobolev embedding that there exists an $(\boldsymbol{u}, \mathbf{T})$ and a (sub)sequence that we do not relabel such that

$$
\begin{aligned}
& \mathbf{T}^{n} \rightarrow^{*} \overline{\mathbf{T}} \\
& \boldsymbol{u}^{n} \rightarrow \boldsymbol{u} \\
& \boldsymbol{u}^{n} \rightarrow \boldsymbol{u} \\
& \frac{\left(\mathbf{T}^{d}\right)^{n}}{n\left|\left(\mathbf{T}^{d}\right)^{n}\right|^{1-\frac{1}{n}}} \rightarrow \mathbf{0} \\
& \frac{\operatorname{tr} \mathbf{T}^{n}}{n\left|\operatorname{tr} \mathbf{T}^{n}\right|^{1-\frac{1}{n}}} \rightarrow \mathbf{0}
\end{aligned}
$$

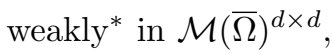

$$
\begin{aligned}
& \text { weakly in } W_{0}^{1,2 d}(\Omega)^{d} \text {, } \\
& \text { strongly in } \mathcal{C}(\Omega)^{d} \text {, } \\
& \text { strongly in } L^{1}(\Omega)^{d \times d} \text {, } \\
& \text { strongly in } L^{1}(\Omega)^{d \times d} \text {. }
\end{aligned}
$$

Thus, we can let $n \rightarrow \infty$ in (67) to deduce that

$$
\langle\overline{\mathbf{T}}, \boldsymbol{\varepsilon}(\boldsymbol{w})\rangle_{\mathcal{M}(\bar{\Omega}), \mathcal{C}(\bar{\Omega})}=\int_{\Omega} \mathbf{F} \cdot \boldsymbol{\varepsilon}(\boldsymbol{w}) \mathrm{d} x \quad \text { for all } \boldsymbol{w} \in \mathcal{D}(\Omega)^{d} .
$$


In addition, using (88) we get that, for all $\Omega_{0} \subset \bar{\Omega}_{0} \subset \Omega$,

$$
\mathbf{T}^{n} \rightarrow \mathbf{T} \quad \text { weakly in } L^{1+\frac{p}{2}}\left(\Omega_{0}\right)^{d \times d} .
$$

Moreover, from the uniqueness of the weak limit, we immediately have that

$$
\overline{\mathbf{T}}=\mathbf{T} \quad \text { almost everywhere in } \Omega,
$$

and because of the choice of the space for the test functions we can replace $\overline{\mathbf{T}}$ by $\mathbf{T}$ in (94) to get (53). In addition, it follows from (75), (86) and the weak lower semicontinuity of the norm that

$$
\boldsymbol{\varepsilon}(\boldsymbol{u}) \in L^{\infty}(\Omega)^{d \times d}, \quad \mathbf{T}^{d} \in L_{l o c}^{\frac{d(1-\alpha)}{d-\beta}}(\Omega) .
$$

Next, we define

and by using (88) we get

$$
\Omega_{k}^{n}:=\left\{x \in \Omega ; \kappa+\left|\left(\mathbf{T}^{d}\right)^{n}\right|+\left|\mathbf{T}^{d}\right|>k\right\}
$$

$$
\left|\Omega_{k}^{n} \cap \Omega_{0}\right| \leq C\left(\Omega_{0}\right) k^{-\frac{p+2}{2}} .
$$

We then use such a decomposition of $\Omega_{0}$ to deduce (denoting by $\tau \in \mathcal{D}(\Omega)$ a nonnegative function such that $\tau \equiv 1$ in $\Omega_{0}$ ) that

$$
\begin{aligned}
& \left(\int_{\Omega_{0}}\left|\left(\mathbf{T}^{d}\right)^{n}-\mathbf{T}^{d}\right| \mathrm{d} x\right)^{2} \\
& =\left(\int_{\Omega_{0} \cap \Omega_{k}^{n}}\left|\left(\mathbf{T}^{d}\right)^{n}-\mathbf{T}^{d}\right| \mathrm{d} x+\int_{\Omega_{0} \backslash \Omega_{k}^{n}}\left|\left(\mathbf{T}^{d}\right)^{n}-\mathbf{T}^{d}\right| \mathrm{d} x\right)^{2} \\
& \leq C\left\|\mathbf{T}^{n}-\mathbf{T}\right\|_{L^{1+\frac{p}{2}}\left(\Omega_{0}\right)}^{2}\left|\Omega_{k}^{n} \cap \Omega_{0}\right|^{\frac{2 p}{p+2}} \\
& +C k^{1+\alpha} \int_{\Omega_{0} \backslash \Omega_{k}^{n}} \frac{\left|\left(\mathbf{T}^{d}\right)^{n}-\mathbf{T}^{d}\right|^{2}}{\left(\kappa+\left|\left(\mathbf{T}^{d}\right)^{n}\right|+\left|\mathbf{T}^{d}\right|\right)^{1+\alpha}} \mathrm{d} x \\
& \leq \frac{C\left(\Omega_{0}\right)}{k^{p}}+C k^{1+\alpha} \int_{\Omega}\left(\lambda\left(\operatorname{tr} \mathbf{T}^{n}\right) \operatorname{tr} \mathbf{T}^{n}-\lambda(\operatorname{tr} \mathbf{T}) \operatorname{tr} \mathbf{T}\right) \cdot\left(\mathbf{T}^{n}-\mathbf{T}\right) \tau \mathrm{d} x \\
& +C k^{1+\alpha} \int_{\Omega}\left(\mu\left(\left|\left(\mathbf{T}^{d}\right)^{n}\right|\right)\left(\mathbf{T}^{d}\right)^{n}-\mu\left(\left|\left(\mathbf{T}^{d}\right)\right|\right)\left(\mathbf{T}^{d}\right)\right) \cdot\left(\mathbf{T}^{n}-\mathbf{T}\right) \tau \mathrm{d} x,
\end{aligned}
$$

where in the last inequality we used the nonnegativity of all terms appearing in the integrals and Lemma 4.1. Thus, using (68) and substituting the corresponding terms by $\boldsymbol{\varepsilon}\left(\boldsymbol{u}^{n}\right)$ we get

$$
\begin{aligned}
& \left\|\left(\mathbf{T}^{d}\right)^{n}-\mathbf{T}^{d}\right\|_{L^{1}\left(\Omega_{0}\right)}^{2} \leq \frac{C\left(\Omega_{0}\right)}{k^{p}} \\
& +C k^{1+\alpha} \int_{\Omega}\left(\varepsilon\left(\boldsymbol{u}^{n}\right)-\frac{\left(\operatorname{tr} \mathbf{T}^{n}\right) \mathbf{I}}{n\left|\operatorname{tr} \mathbf{T}^{n}\right|^{1-\frac{1}{n}}}-\frac{\left(\mathbf{T}^{n}\right)^{d}}{n\left|\left(\mathbf{T}^{n}\right)^{d}\right|^{1-\frac{1}{n}}}\right) \cdot\left(\mathbf{T}^{n}-\mathbf{T}\right) \tau \mathrm{d} x \\
& -C k^{1+\alpha} \int_{\Omega}\left(\mu\left(\left|\left(\mathbf{T}^{d}\right)\right|\right)\left(\mathbf{T}^{d}\right)+\lambda(\operatorname{tr} \mathbf{T})(\operatorname{tr} \mathbf{T}) \mathbf{I}\right) \cdot\left(\mathbf{T}^{n}-\mathbf{T}\right) \tau \mathrm{d} x .
\end{aligned}
$$

Thus, letting $n \rightarrow \infty$, using (74), (75), (67), (94), (91) and the fact that $\tau$ is compactly supported in $\Omega$ we deduce that

$$
\begin{aligned}
& \lim _{n \rightarrow \infty}\left\|\left(\mathbf{T}^{d}\right)^{n}-\mathbf{T}^{d}\right\|_{L^{1}\left(\Omega_{0}\right)}^{2} \leq \frac{C\left(\Omega_{0}\right)}{k^{p}}+C k^{1+\alpha} \lim _{n \rightarrow \infty} \int_{\Omega} \varepsilon\left(\boldsymbol{u}^{n}\right) \cdot\left(\mathbf{T}^{n}-\mathbf{T}\right) \tau \mathrm{d} x \\
& =\frac{C\left(\Omega_{0}\right)}{k^{p}}+C k^{1+\alpha} \lim _{n \rightarrow \infty} \int_{\Omega}\left(\varepsilon\left(\boldsymbol{u}^{n} \tau\right)-\boldsymbol{u}^{n} \otimes \nabla \tau\right) \cdot\left(\mathbf{T}^{n}-\mathbf{T}\right) \mathrm{d} x \\
& =\frac{C\left(\Omega_{0}\right)}{k^{p}}-C k^{1+\alpha} \lim _{n \rightarrow \infty} \int_{\Omega}\left(\boldsymbol{u}^{n} \otimes \nabla \tau\right) \cdot\left(\mathbf{T}^{n}-\mathbf{T}\right) \mathrm{d} x=\frac{C\left(\Omega_{0}\right)}{k^{p}} \stackrel{k \rightarrow \infty}{\rightarrow} 0 .
\end{aligned}
$$

Consequently, using also (95), we see that

$$
\left(\mathbf{T}^{d}\right)^{n} \rightarrow \mathbf{T}^{d} \quad \text { strongly in } L^{q}\left(\Omega_{0}\right)^{d \times d} \text { for any } q \in\left[1,1+\frac{p}{2}\right) .
$$


Finally, combining (67) and (59), we also have the following inequality

$$
\left\|\mathbf{T}^{n}-\mathbf{T}\right\|_{L^{q}\left(\Omega_{0}\right)}^{q} \leq C\left\|\left(\mathbf{T}^{d}\right)^{n}-\mathbf{T}^{d}\right\|_{L^{q}\left(\Omega_{0}\right)}^{q}+C\left|\int_{\Omega_{0}} \operatorname{tr} \mathbf{T}^{n}-\operatorname{tr} \mathbf{T} \mathrm{d} x\right|^{q},
$$

which, after using (95) and (101), leads to the conclusion that

$$
\mathbf{T}^{n} \rightarrow \mathbf{T} \quad \text { strongly in } L^{q}\left(\Omega_{0}\right)^{d \times d} \text { for any } q \in\left[1,1+\frac{p}{2}\right),
$$

and consequently, since $\Omega_{0}$ is arbitrary, we see that we can find a subsequence such that

$$
\mathbf{T}^{n} \rightarrow \mathbf{T} \quad \text { a.e. in } \Omega \text {. }
$$

Using Fatou's lemma, (74) and (104), we then have that

$$
\int_{\Omega}|\mathbf{T}| \mathrm{d} x \leq C
$$

and, by invoking the strong convergence result (104), we can let $n \rightarrow \infty$ in (106) to deduce that

$$
\boldsymbol{\varepsilon}(\boldsymbol{u})=\lambda(\operatorname{tr} \mathbf{T})(\operatorname{tr} \mathbf{T}) \mathbf{I}+\mu\left(\left|\mathbf{T}^{d}\right|\right) \mathbf{T}^{d} \quad \text { in } \Omega .
$$

Consequently, using (A1)-(A2), we see that

$$
\|\varepsilon(\boldsymbol{u})\|_{\infty} \leq C
$$

Thus, the proof is complete.

4.3. Regularity. This section is devoted to establishing higher regularity of the solution to (45). For this purpose, we introduce a slightly different approximation than in the previous subsection, which will lead to a problem in a Hilbert space setting, for which we can use standard theory. Note that such a procedure would have failed in the previous subsection because of the low regularity assumed on $\mathbf{F}$. Hence, for any fixed $n \in \mathbb{N}$ we consider the problem

$$
\begin{aligned}
\int_{\Omega} \mathbf{T} \cdot \boldsymbol{\varepsilon}(\boldsymbol{w}) \mathrm{d} x & =\int_{\Omega} \boldsymbol{f} \cdot \boldsymbol{w} \mathrm{d} x & & \text { for all } \boldsymbol{w} \in W_{0}^{1,2}(\Omega)^{d}, \\
\varepsilon(\boldsymbol{u}) & =\lambda(\operatorname{tr} \mathbf{T})(\operatorname{tr} \mathbf{T}) \mathbf{I}+\mu\left(\left|\mathbf{T}^{d}\right|\right) \mathbf{T}^{d}+\frac{1}{n} \mathbf{T} & & \text { in } \Omega, \\
\boldsymbol{u} & =\mathbf{0} & & \text { on } \partial \Omega .
\end{aligned}
$$

Similarly as in the previous subsection, we see that for fixed $n$ the identity (106) can be equivalently rewritten as

$$
\mathbf{T}=\mathbf{T}^{*}(\varepsilon(\boldsymbol{u}))
$$

where $\mathbf{T}^{*}$ is a uniformly monotone mapping having at most linear growth at infinity. Therefore, the existence and uniqueness of the solution $\boldsymbol{u}_{n} \in W_{0}^{1,2}(\Omega)^{d}$ and $\mathbf{T}_{n} \in L^{2}(\Omega)^{d \times d}$ to (105)-(107) follows from standard monotone operator theory. Moreover, thanks to the uniform monotonicity of the nonlinearity, we can apply the difference quotient technique considered in the previous section to show that $\nabla^{2} \boldsymbol{u}_{n}$ and $\nabla \mathbf{T}_{n}$ belong to $L_{l o c}^{2}(\Omega)^{d \times d \times d}$.

Initial a priori $n$-independent estimates. For simplicity we omit writing the subscript $n$ in this subsection and we shall denote by $C>0$ a generic positive constant, which may change from line to line, and which depends only on the data but not on $n$. We begin by establishing some initial a priori estimates. Thus, setting $\boldsymbol{w}:=\boldsymbol{u}$ in (105) and using the symmetry of $\mathbf{T}$, we get the identity

$$
\int_{\Omega} \mathbf{T} \cdot \boldsymbol{\varepsilon}(\boldsymbol{u}) \mathrm{d} x=\int_{\Omega} \boldsymbol{f} \cdot \boldsymbol{u} \mathrm{d} x,
$$

which, after using (106) to eliminate $\boldsymbol{\varepsilon}(\boldsymbol{u})$, gives the identity

$$
\int_{\Omega} \lambda(\operatorname{tr} \mathbf{T})|\operatorname{tr} \mathbf{T}|^{2}+\mu\left(\left|\mathbf{T}^{d}\right|\right)\left|\mathbf{T}^{d}\right|^{2}+\frac{1}{n}|\mathbf{T}|^{2} \mathrm{~d} x=\int_{\Omega} \boldsymbol{f} \cdot \boldsymbol{u} \mathrm{d} x .
$$


It then follows from (A1) and (A2) and Hölder's and Korn's inequalities that

$$
\int_{\Omega}|\mathbf{T}|+\frac{1}{n}|\mathbf{T}|^{2} \leq C\left(1+\|\boldsymbol{f}\|_{2}\|\varepsilon(\boldsymbol{u})\|_{2}\right)
$$

To bound the term on the right-hand side, we again use (106), (A1) and (A2) to observe that

$$
|\varepsilon(\boldsymbol{u})| \leq 2 C_{2}+\frac{1}{n}|\mathbf{T}|
$$

which implies that

$$
\|\varepsilon(\boldsymbol{u})\|_{2} \leq C+\frac{C}{n}\|\mathbf{T}\|_{2}
$$

Hence, substituting (110) into (108) and using Young's and Korn's inequalities, we deduce the uniform bound

$$
\|\boldsymbol{u}\|_{1,2}^{2}+\|\mathbf{T}\|_{1}+\frac{\|\mathbf{T}\|_{2}^{2}}{n} \leq C\left(1+\|\boldsymbol{f}\|_{2}^{2}\right)
$$

Differential inequality for $\nabla \mathbf{T}$. The next step is to improve the estimates in the interior of $\Omega$. Since for the approximating problem we have sufficient regularity, we can consider the equation (105) pointwise, i.e.,

$$
-\sum_{j=1}^{d} D_{j} \mathbf{T}_{i j}=\boldsymbol{f}_{i}, \quad \text { a.e. in } \Omega, \quad i=1, \ldots, d
$$

where we denoted $D_{j}:=\frac{\partial}{\partial x_{j}}$. Next, we apply $D_{k}$ (of course we understand $D_{k}$ as a weak derivative) to (112), multiply by $D_{k} \boldsymbol{u}_{i}$ and sum with respect to $i$ and $k$ to get

$$
-\sum_{i, j, k=1}^{d} D_{j k} \mathbf{T}_{i j} D_{k} \boldsymbol{u}_{i}=\nabla \boldsymbol{f} \cdot \nabla \boldsymbol{u} \quad \text { in } \Omega .
$$

The term on the left-hand side is the source of additional information and we therefore rewrite it as follows:

$$
\begin{aligned}
\sum_{i, j, k=1}^{d} D_{j k} \mathbf{T}_{i j} D_{k} \boldsymbol{u}_{i}= & \sum_{i, j, k=1}^{d} D_{j}\left(D_{k} \mathbf{T}_{i j} D_{k} \boldsymbol{u}_{i}\right)-\sum_{i, j, k=1}^{d} D_{k} \mathbf{T}_{i j} D_{j k} \boldsymbol{u}_{i} \\
= & 2 \sum_{i, j, k=1}^{d} D_{j}\left(D_{k} \mathbf{T}_{i j} \varepsilon_{i k}(\boldsymbol{u})\right)-\sum_{i, j, k=1}^{d} D_{j}\left(D_{k} \mathbf{T}_{i j} D_{i} \boldsymbol{u}_{k}\right) \\
& -\nabla \mathbf{T} \cdot \nabla \boldsymbol{\varepsilon}(\boldsymbol{u})
\end{aligned}
$$

where for the second equality we used the symmetry of $\mathbf{T}$ and the definition of $\boldsymbol{\varepsilon}(\boldsymbol{u})$. Next, for the second term on the right-hand side we use the following relation

$$
D_{i j} \boldsymbol{u}_{k}=D_{j} \varepsilon_{i k}(\boldsymbol{u})+D_{i} \varepsilon_{j k}(\boldsymbol{u})-D_{k} \boldsymbol{\varepsilon}_{i j}(\boldsymbol{u})
$$


to be able to replace $\nabla \boldsymbol{u}$ by $\boldsymbol{\varepsilon}(\boldsymbol{u})$, which then yields

$$
\begin{aligned}
& \sum_{i, j, k=1}^{d} D_{j}\left(D_{k} \mathbf{T}_{i j} D_{i} \boldsymbol{u}_{k}\right)=\sum_{i, j, k=1}^{d} D_{j k}\left(\mathbf{T}_{i j} D_{i} \boldsymbol{u}_{k}\right)-\sum_{i, j, k=1}^{d} D_{j}\left(\mathbf{T}_{i j} D_{i k} \boldsymbol{u}_{k}\right) \\
= & \sum_{i, j, k=1}^{d} D_{k}\left(D_{j} \mathbf{T}_{i j} D_{i} \boldsymbol{u}_{k}\right)+\sum_{i, j, k=1}^{d} D_{k}\left(\mathbf{T}_{i j} D_{i j} \boldsymbol{u}_{k}\right)-\sum_{i, j=1}^{d} D_{j}\left(\mathbf{T}_{i j} D_{i} \operatorname{div} \boldsymbol{u}\right) \\
= & -\sum_{i, k=1}^{d} D_{k}\left(\boldsymbol{f}_{i} D_{i} \boldsymbol{u}_{k}\right)+\sum_{i, j, k=1}^{d} D_{k}\left(\mathbf{T}_{i j}\left(D_{j} \varepsilon_{i k}(\boldsymbol{u})+D_{i} \boldsymbol{\varepsilon}_{j k}(\boldsymbol{u})-D_{k} \boldsymbol{\varepsilon}_{i j}(\boldsymbol{u})\right)\right) \\
& -\sum_{i, j=1}^{d} D_{i j}\left(\mathbf{T}_{i j} \operatorname{div} \boldsymbol{u}\right)+\sum_{i, j=1}^{d} D_{j}\left(D_{i} \mathbf{T}_{i j} \operatorname{div} \boldsymbol{u}\right) \\
= & -\sum_{i, k=1}^{d} D_{k}\left(\boldsymbol{f}_{i} D_{i} \boldsymbol{u}_{k}\right)+2 \sum_{i, j, k=1}^{d} D_{j k}\left(\mathbf{T}_{i j} \varepsilon_{i k}(\boldsymbol{u})\right)-\triangle(\mathbf{T} \cdot \boldsymbol{\varepsilon}(\boldsymbol{u})) \\
& +2 \sum_{i, k=1}^{d} D_{k}\left(\boldsymbol{f}_{i} \boldsymbol{\varepsilon}_{i k}(\boldsymbol{u})\right)+\sum_{i, j, k=1}^{d} D_{k}\left(D_{k} \mathbf{T}_{i j} \boldsymbol{\varepsilon}_{i j}(\boldsymbol{u})\right) \\
& -\sum_{i, j=1}^{d} D_{i j}\left(\mathbf{T}_{i j} \operatorname{tr} \boldsymbol{\varepsilon}(\boldsymbol{u})\right)-\sum_{j=1}^{d} D_{j}\left(\boldsymbol{f}_{j} \operatorname{tr} \boldsymbol{\varepsilon}(u)\right)
\end{aligned}
$$

where the identity (112) was used to replace div T. Hence, using this expression in (114) we can simply write

$$
-\sum_{i, j, k=1}^{d} D_{j k} \mathbf{T}_{i j} D_{k} \boldsymbol{u}_{i}=\nabla \mathbf{T} \cdot \nabla \boldsymbol{\varepsilon}(u)-\operatorname{div} \operatorname{div} \mathbf{H}-\operatorname{div} \boldsymbol{v}
$$

where

$$
\begin{aligned}
\mathbf{H}_{i j} & :=-2 \sum_{k=1}^{d} \mathbf{T}_{i k} \boldsymbol{\varepsilon}_{j k}(\boldsymbol{u})+\delta_{i j} \mathbf{T} \cdot \boldsymbol{\varepsilon}(\boldsymbol{u})+\operatorname{tr} \boldsymbol{\varepsilon}(\boldsymbol{u}) \mathbf{T}_{i j}, \\
\boldsymbol{v}_{i} & :=2 \sum_{j, k=1}^{d} D_{k} \mathbf{T}_{i j} \boldsymbol{\varepsilon}_{j k}(u)-\sum_{j=1}^{d} \boldsymbol{f}_{j} D_{i} \boldsymbol{u}_{j}+\operatorname{tr} \boldsymbol{\varepsilon}(\boldsymbol{u}) \boldsymbol{f}_{j}-\sum_{j, k=1}^{d} D_{i} \mathbf{T}_{j k} \boldsymbol{\varepsilon}_{j k}(\boldsymbol{u}) .
\end{aligned}
$$

Finally, (113) then directly implies the inequality

$$
\nabla \mathbf{T} \cdot \nabla \boldsymbol{\varepsilon}(\boldsymbol{u}) \leq|\nabla \boldsymbol{f}||\nabla \boldsymbol{u}|+\operatorname{div} \operatorname{div} \mathbf{H}+\operatorname{div} \boldsymbol{v}
$$

Moreover, using the definitions (117)-(118) and the estimate (109), we immediately obtain the following bounds on the 'pollution terms' appearing on the right-hand side of (119):

$$
\begin{aligned}
& |\mathbf{H}| \leq C|\mathbf{T}|+\frac{C|\mathbf{T}|^{2}}{n} \\
& |\boldsymbol{v}| \leq C\left(|\boldsymbol{f}||\nabla \boldsymbol{u}|+|\nabla \mathbf{T}|+\frac{|\nabla \mathbf{T}||\mathbf{T}|}{n}\right) .
\end{aligned}
$$


The last step of this subsection is to provide a suitable lower bound on the term on the left-hand side of (119). Thus, using the definition (106) and the assumptions (A1)-(A4) we get

$$
\begin{aligned}
\nabla \mathbf{T} \cdot \nabla \boldsymbol{\varepsilon}(\boldsymbol{u})= & \frac{|\nabla \mathbf{T}|^{2}}{n}+\nabla(\lambda(\operatorname{tr} \mathbf{T})(\operatorname{tr} \mathbf{T})) \cdot \nabla(\operatorname{tr} \mathbf{T})+\nabla\left(\mu\left(\left|\mathbf{T}^{d}\right|\right) \mathbf{T}^{d}\right) \cdot \nabla \mathbf{T}^{d} \\
= & \frac{|\nabla \mathbf{T}|^{2}}{n}+\left(\lambda^{\prime}(\operatorname{tr} \mathbf{T})(\operatorname{tr} \mathbf{T})+\lambda(\operatorname{tr} \mathbf{T})\right)|\nabla| \operatorname{tr} \mathbf{T} \|^{2} \\
& +\mu\left(\left|\mathbf{T}^{d}\right|\right)\left|\nabla \mathbf{T}^{d}\right|^{2}+\mu^{\prime}\left(\left|\mathbf{T}^{d}\right|\right)\left|\mathbf{T}^{d}\right||\nabla| \mathbf{T}^{d}||^{2} \\
\geq & \frac{|\nabla \mathbf{T}|^{2}}{n}+\left.|\nabla| \operatorname{tr} \mathbf{T}\right|^{2}(\lambda(s) s)_{s=\operatorname{tr} \mathbf{T}}^{\prime} \\
& +\left|\nabla \mathbf{T}^{d}\right|^{2} \min \left\{\mu\left(\left|\mathbf{T}^{d}\right|\right),(\mu(s) s)_{s=\left|\mathbf{T}^{d}\right|}^{\prime}\right\} \\
\geq & \frac{|\nabla \mathbf{T}|^{2}}{n}+\frac{C_{1}\left|\nabla \mathbf{T}^{d}\right|^{2}}{\left(\kappa+\left|\mathbf{T}^{d}\right|\right)^{\alpha+1}}
\end{aligned}
$$

Consequently, using this inequality in (119) we obtain the final relation

$$
\frac{|\nabla \mathbf{T}|^{2}}{n}+\frac{C_{1}\left|\nabla \mathbf{T}^{d}\right|^{2}}{\left(\kappa+\left|\mathbf{T}^{d}\right|\right)^{\alpha+1}} \leq|\nabla \boldsymbol{f}||\nabla \boldsymbol{u}|+\operatorname{div} \operatorname{div} \mathbf{H}+\operatorname{div} \boldsymbol{v} .
$$

Interior uniform estimates for $\nabla \mathbf{T}$. This subsection is devoted to the essential estimates that will be crucial when letting $n \rightarrow \infty$. Hence, let $\eta \in \mathcal{D}(\Omega)$ be an arbitrary nonnegative function with $\|\eta\|_{\infty} \leq 1$. For some $k \in \mathbb{N}$ that will be specified later, we multiply (123) by $\eta^{2 k}$, integrate over $\Omega$ and use integration by parts to obtain

$$
\begin{aligned}
& \int_{\Omega} \frac{|\nabla \mathbf{T}|^{2} \eta^{2 k}}{n}+\frac{C_{1}\left|\nabla \mathbf{T}^{d}\right|^{2} \eta^{2 k}}{\left(\kappa+\left|\mathbf{T}^{d}\right|\right)^{\alpha+1}} \mathrm{~d} x \\
& \quad \leq \int_{\Omega}|\nabla \boldsymbol{f} \| \nabla \boldsymbol{u}| \eta^{2 k}+|\mathbf{H}|\left|\nabla^{2} \eta^{2 k}\right|+|\boldsymbol{v}|\left|\nabla \eta^{2 k}\right| \mathrm{d} x
\end{aligned}
$$

Then, using the estimates (120)-(121) we get (here $C(k, \eta)$ denotes a generic positive constant depending only on $k$ and $\eta$ ):

$$
\begin{aligned}
& \int_{\Omega} \frac{|\nabla \mathbf{T}|^{2} \eta^{2 k}}{n}+\frac{\left|\nabla \mathbf{T}^{d}\right|^{2} \eta^{2 k}}{\left(\kappa+\left|\mathbf{T}^{d}\right|\right)^{\alpha+1}} \mathrm{~d} x \\
& \leq C(\eta, k) \int_{\Omega}(|\nabla \boldsymbol{f}|+|\boldsymbol{f}|)|\nabla \boldsymbol{u}|+|\mathbf{T}|+\frac{|\mathbf{T}|^{2}}{n} \mathrm{~d} x \\
& \quad+C(\eta, k) \int_{\Omega}\left(|\nabla \mathbf{T}| \eta^{k}\right)\left(1+\frac{|\mathbf{T}|}{n}\right) \eta^{k-1} \mathrm{~d} x \\
& \leq C(\eta, k) \int_{\Omega}(|\nabla \boldsymbol{f}|+|\boldsymbol{f}|)|\nabla \boldsymbol{u}|+|\mathbf{T}|+\frac{|\mathbf{T}|^{2}}{n} \mathrm{~d} x \\
& \quad+C(\eta, k) \int_{\Omega}|\nabla \mathbf{T}| \eta^{2 k-1} \mathrm{~d} x+\int_{\Omega} \frac{|\nabla \mathbf{T}|^{2} \eta^{2 k}}{n} \mathrm{~d} x
\end{aligned}
$$

where for the second inequality we used Young's inequality and the fact that $k \geq 1$. Note that while the last term on the right-hand side can be absorbed into the left-hand side, for the first integral we can use the uniform bound (111) and then the estimate (125) reduces to

$$
\int_{\Omega} \frac{\left|\nabla \mathbf{T}^{d}\right|^{2} \eta^{2 k}}{\left(\kappa+\left|\mathbf{T}^{d}\right|\right)^{\alpha+1}} \mathrm{~d} x \leq C(\eta, k, \boldsymbol{f})+C(\eta, k) \int_{\Omega}|\nabla \mathbf{T}| \eta^{2 k-1} \mathrm{~d} x .
$$


Thus, using (60) in (126), the relation (112) and Young's inequality we get

$$
\begin{aligned}
& \int_{\Omega} \frac{\left|\nabla \mathbf{T}^{d}\right|^{2} \eta^{2 k}}{\left(\kappa+\left|\mathbf{T}^{d}\right|\right)^{\alpha+1}} \mathrm{~d} x \leq C(\eta, k, \boldsymbol{f})+C(\eta, k) \int_{\Omega}\left|\nabla \mathbf{T}^{d}\right| \eta^{2 k-1} \mathrm{~d} x \\
& \quad=C(\eta, k, \boldsymbol{f})+C(\eta, k) \int_{\Omega}\left(\frac{\left|\nabla \mathbf{T}^{d}\right|^{2} \eta^{2 k}}{\left(\kappa+\left|\mathbf{T}^{d}\right|\right)^{\alpha+1}}\right)^{\frac{1}{2}}\left(\kappa+\left|\mathbf{T}^{d}\right|\right)^{\frac{\alpha+1}{2}} \eta^{k-1} \mathrm{~d} x \\
& \quad \leq C(\eta, k, \boldsymbol{f})+\frac{1}{2} \int_{\Omega} \frac{\left|\nabla \mathbf{T}^{d}\right|^{2} \eta^{2 k}}{\left(\kappa+\left|\mathbf{T}^{d}\right|\right)^{\alpha+1}} \mathrm{~d} x+C(\eta, k) \int_{\Omega}\left(\kappa+\left|\mathbf{T}^{d}\right|\right)^{\alpha+1} \eta^{2 k-2} \mathrm{~d} x
\end{aligned}
$$

Hence, by a simple algebraic manipulation, also

$$
\int_{\Omega} \frac{\left|\nabla \mathbf{T}^{d}\right|^{2} \eta^{2 k}}{\left(\kappa+\left|\mathbf{T}^{d}\right|\right)^{\alpha+1}} \mathrm{~d} x \leq C(\eta, k, \boldsymbol{f})+C(\eta, k) \int_{\Omega}\left(\kappa+\left|\mathbf{T}^{d}\right|\right)^{\alpha+1} \eta^{2 k-2} \mathrm{~d} x .
$$

Next, we shall use the embedding theorem to extract further information from the term on the left-hand side. Before doing so, we use the term on the left-hand side for the following estimate:

$$
\left|\nabla\left(\left(\kappa+\left|\mathbf{T}^{d}\right|\right)^{\frac{1-\alpha}{2}} \eta^{k}\right)\right|^{2} \leq C \frac{\left|\nabla \mathbf{T}^{d}\right|^{2} \eta^{2 k}}{\left(\kappa+\left|\mathbf{T}^{d}\right|\right)^{\alpha+1}}+C\left(\kappa+\left|\mathbf{T}^{d}\right|\right)^{1-\alpha}\left|\nabla \eta^{k}\right|^{2},
$$

which, after integration over $\Omega$ and using the uniform bound (111), leads to

$$
\int_{\Omega}\left|\nabla\left(\left(\kappa+\left|\mathbf{T}^{d}\right|\right)^{\frac{1-\alpha}{2}} \eta^{k}\right)\right|^{2} \mathrm{~d} x \leq C(\eta, k, \boldsymbol{f})+C \int_{\Omega} \frac{\left|\nabla \mathbf{T}^{d}\right|^{2} \eta^{2 k}}{\left(\kappa+\left|\mathbf{T}^{d}\right|\right)^{\alpha+1}} \mathrm{~d} x .
$$

Hence, combining this with (127) and using the presence of the cut-off function we get

$$
\begin{aligned}
& \left\|\left(\kappa+\left|\mathbf{T}^{d}\right|\right)^{\frac{1-\alpha}{2}} \eta^{k}\right\|_{1,2}^{2} \leq C(\eta, k, \boldsymbol{f})+C(\eta, k) \int_{\Omega}\left(\kappa+\left|\mathbf{T}^{d}\right|\right)^{\alpha+1} \eta^{2 k-2} \mathrm{~d} x \\
& =C(\eta, k, \boldsymbol{f})+C(\eta, k) \int_{\Omega}\left(\left(\kappa+\left|\mathbf{T}^{d}\right|\right)^{\frac{1-\alpha}{2}} \eta^{k}\right)^{\frac{2(k-1)}{k}}\left(\kappa+\left|\mathbf{T}^{d}\right|\right)^{2 \alpha+\frac{1-\alpha}{k}} \mathrm{~d} x \\
& \leq C(\eta, k, \boldsymbol{f})+C(\eta, k)\left\|\kappa+\left|\mathbf{T}^{d}\right|\right\|_{1}^{2 \alpha+\frac{1-\alpha}{k}}\left\|\left(\kappa+\left|\mathbf{T}^{d}\right|\right)^{\frac{1-\alpha}{2}} \eta^{k}\right\|_{\frac{2(k-1)}{k\left(1-2 \alpha-\frac{1-\alpha}{k}\right)}}^{\frac{2(k-1)}{k\left(1-\frac{1-\alpha}{k}\right.}}
\end{aligned}
$$

where we have assumed that $k$ is chosen sufficiently large, so as to ensure that

$$
2 \alpha+\frac{1-\alpha}{k}<1
$$

Note that such a choice is always possible because of our assumptions on $\alpha$; namely, we know that $\alpha<\frac{1}{2}$. Thus, using (111), (129) reduces to

$$
\left\|\left(\kappa+\left|\mathbf{T}^{d}\right|\right)^{\frac{1-\alpha}{2}} \eta^{k}\right\|_{1,2}^{2} \leq C(\eta, k, \boldsymbol{f})+C(\eta, k)\left\|\left(\kappa+\left|\mathbf{T}^{d}\right|\right)^{\frac{1-\alpha}{2}} \eta^{k}\right\|_{\frac{2(k-1)}{k\left(1-2 \alpha-\frac{1-\alpha}{k}\right)}}^{\frac{2(k-1)}{k}} .
$$

Finally, if we are able to find a sufficiently large $k$ such that

$$
W^{1,2}(\Omega) \hookrightarrow L^{\frac{2(k-1)}{k\left(1-2 \alpha-\frac{1-\alpha}{k}\right)}}(\Omega),
$$

then we can use Young's inequality to deduce that

$$
\left\|\left(\kappa+\left|\mathbf{T}^{d}\right|\right)^{\frac{1-\alpha}{2}} \eta^{k}\right\|_{1,2}^{2} \leq C(\eta, k, \boldsymbol{f}) .
$$

Hence, it remains to discuss (132). First, if $d=2$, then it suffices to consider $k$ fulfilling (130). For $d \geq 3$ we need to show that

$$
\frac{2 d}{d-2} \geq \frac{2(k-1)}{k\left(1-2 \alpha-\frac{1-\alpha}{k}\right)} .
$$

Since the right-hand side is increasing with respect to $k$ it is enough to show that

$$
\frac{2 d}{d-2}>\frac{2}{1-2 \alpha} \quad \Leftrightarrow \quad \alpha<\frac{1}{d},
$$

which is nothing else than our assumption on $\alpha$. 
Having obtained the uniform, $n$-independent, bound (133), we can use the embedding theorem to deduce that for any $\Omega_{0} \subset \bar{\Omega}_{0} \subset \Omega$ we have (if $d=2$ we replace the exponent by any $q<\infty$ )

$$
\int_{\Omega_{0}}\left|\mathbf{T}^{d}\right|^{\frac{d(1-\alpha)}{d-2}} \mathrm{~d} x \leq C\left(\Omega_{0}\right) .
$$

Moreover, since $\frac{d(1-\alpha)}{d-2} \geq \alpha+1$ we deduce from (127) that

$$
\int_{\Omega_{0}} \frac{\left|\nabla \mathbf{T}^{d}\right|^{2}}{\left(\kappa+\left|\mathbf{T}^{d}\right|\right)^{1+\alpha}} \mathrm{d} x \leq C\left(\Omega_{0}\right) .
$$

Finally, for any $q \in[1,2)$, Young's inequality implies that

$$
\left|\nabla \mathbf{T}^{d}\right|^{q}=\left(\frac{\left|\nabla \mathbf{T}^{d}\right|^{2}}{\left(\kappa+\left|\mathbf{T}^{d}\right|\right)^{1+\alpha}}\right)^{\frac{q}{2}}\left(\kappa+\left|\mathbf{T}^{d}\right|\right)^{\frac{q(1+\alpha)}{2}} \leq \frac{\left|\nabla \mathbf{T}^{d}\right|^{2}}{\left(\kappa+\left|\mathbf{T}^{d}\right|\right)^{1+\alpha}}+\left(\kappa+\left|\mathbf{T}^{d}\right|\right)^{\frac{q(1+\alpha)}{2-q}} .
$$

Thus, integrating over $\Omega_{0}$ and using (135) we get

$$
\int_{\Omega_{0}}\left|\nabla \mathbf{T}^{d}\right|^{q} \mathrm{~d} x \leq C\left(\Omega_{0}\right)+C \int_{\Omega_{0}}\left|\mathbf{T}^{d}\right|^{\frac{q(1+\alpha)}{2-q}} \mathrm{~d} x
$$

and from (60) and (112) we deduce that

$$
\int_{\Omega_{0}}|\nabla \mathbf{T}|^{q} \mathrm{~d} x \leq C\left(\Omega_{0}, \boldsymbol{f}\right)+C \int_{\Omega_{0}}\left|\mathbf{T}^{d}\right|^{\frac{q(1+\alpha)}{2-q}} \mathrm{~d} x .
$$

To bound the term on the right-hand side we use (134) and choose $q$ such that (if $d=2$, then $q \in[1,2)$ can be chosen arbitrarily)

to deduce that

$$
\frac{q(1+\alpha)}{2-q}=\frac{d(1-\alpha)}{d-2} \Leftrightarrow q=\frac{2 d(1-\alpha)}{(d-2)(1+\alpha)+d(1-\alpha)}
$$

$$
\int_{\Omega_{0}}|\nabla \mathbf{T}|^{2-\frac{2(d-2)(1+\alpha)}{(d-2)(1+\alpha)+d(1-\alpha)}} \mathrm{d} x \leq C\left(\Omega_{0}\right) .
$$

This then establishes the desired regularity of $\mathbf{T}$ and completes the proof of the theorem.

\section{Conclusions And open PRoblems}

As we have emphasized at the beginning of the paper, in order to understand nonlinear responses of materials to external stimuli of different sort, it is useful to have at one's disposal a broad spectrum of models that have the capacity to describe in mathematical terms a wide range of material behavior. Our objective was to survey recent developments in the implicit constitutive theory of materials, which is able to capture experimental observations that were previously beyond the reach of mathematical models. Implicit constitutive theory also provides theoretical justification in the full three-dimensional setting for various models that were previously proposed in an ad hoc manner. We have highlighted that while the classical linearization based on the displacement gradient being small implies that the classical Cauchy elastic model reduces to the linearized elastic model wherein the strain and the stress are related linearly, linearization, under the same conditions, of the model in which the large strain is a nonlinear function of stress leads to a possibly nonlinear relationship between the linearized strain and the stress.

From the perspective of the theory of nonlinear partial differential equations, implicit constitutive theory leads to new classes of challenging mathematical problems. This study focused on implicitly constituted models for elastic solids in general, and on its subclass consisting of elastic solids with limiting small strain. After introducing the basic concepts of implicit constitutive theory, we presented an overview of results concerning modeling within the framework of continuum mechanics. We then concentrated on the mathematical analysis of relevant boundary-value problems associated with models with limiting small strain, and we have surveyed the availableanalytical results, some of which have been presented for the first time. In particular, we have established the first analytical result concerning the existence of weak solutions in general threedimensional domains. 
The subject is still in its infancy, and there are therefore numerous relevant open questions concerning modelling, PDE analysis and numerical analysis. Some of these are listed below:

1. Even the particular PDE model studied in Section 4 here gives rise to several open problems, including, for example, the extension of the concept of weak solution to all values of $\alpha>0$; the analysis of mixed Dirichlet-Neumann boundary-value problems; the analysis of the full traction problem; the construction of numerical algorithms for these and their rigorous convergence analysis.

2. Another area of interest are computer simulations and systematic computational studies concerning concentrated loads inside the domain or on the boundary, due to contacts, corners, crack tips, notches, and line singularities (by degeneracy of elliptic holes); see fro example [32].

3. As has been indicated in the Introduction, one could also study these types of responses in more complicated settings, such as the Kelvin-Voigt or 'Maxwell' models (inspired by placing a spring and a wire either in parallel or in series with a dashpot).

4. An interesting and challenging question is the analysis of large strains and of evolutionary problems for implicitly constituted material models.

The PDE analysis and numerical analysis of mathematical models for implicitly constituted fluid flow models has been the subject of active research in recent year; the reader is referred to [7] for the PDE analysis of a Bingham fluid model with threshold slip boundary condition; to [5] for a survey of implicit models and the analysis of the existence of solutions in the case of steady problems; and to [6] for the analysis of time-dependent implicitly constituted fluid flow models. As a first step in the direction of rigorous convergence analysis of finite element approximations to implicitly constituted fluid flow models we refer the reader to the recent work of Diening, Kreuzer and Süli [20].

\section{REFERENCES}

[1] M. Bildhauer and M. Fuchs. Regularity for dual solutions and for weak cluster points of minimizing sequences of variational problems with linear growth. Zap. Nauchn. Sem. S.-Peterburg. Otdel. Mat. Inst. Steklov. (POMI), 259 (Kraev. Zadachi Mat. Fiz. i Smezh. Vopr. Teor. Funkts. 30):46-66, 296, 1999.

[2] M. Bildhauer and M. Fuchs. On a class of variational integrals with linear growth satisfying the condition of -ellipticity. Rend. Mat. Appl. (7), 22:249-274 (2003), 2002.

[3] M. Bildhauer and M. Fuchs. Convex variational problems with linear growth. In Geometric analysis and nonlinear partial differential equations, pages 327-344. Springer, Berlin, 2003.

[4] C. Bridges and K.R. Rajagopal. Implicit constitutive models with a thermodynamic basis: a study of stress concentration. Z. Angew. Math. Phys., online first, 2014. DOI: 10.1007/s00033-014-0398-5.

[5] M. Bulíček, P. Gwiazda, J. Málek, K.R. Rajagopal, and A. Świerczewska-Gwiazda. On flows of fluids described by an implicit constitutive equation characterized by a maximal monotone graph. In Mathematical aspects of fluid mechanics, volume 402 of London Math. Soc. Lecture Note Ser., pages 23-51. Cambridge Univ. Press, Cambridge, 2012.

[6] M. Bulíček, P. Gwiazda, J. Málek, and A. Świerczewska-Gwiazda. On unsteady flows of implicitly constituted incompressible fluids. SIAM J. Math. Anal., 44(4):2756-2801, 2012.

[7] M. Bulíček and J. Málek. On unsteady internal flows of Bingham fluids subject to threshold slip on the impermeable boundary. Preprint MORE/2013/06 - accepted for publication in "Recent Developments of Mathematical Fluid Mechanics", series: Advances in Mathematical Fluid Mechanics, Birkhauser-Verlag, 2014.

[8] M. Bulíček, J. Málek, K.R. Rajagopal, and J. Walton. Existence of solutions for the anti-plane stress for a new class of "strain-limiting" elastic bodies. Submitted for publication in Calc. Var. and PDE, 2014.

[9] M. Bulíček, J. Málek, and E. Süli. Analysis and approximation of a strain-limiting nonlinear elastic model. To appear in Mathematics and Mechanics of Solids, 2014.

[10] J.M. Burgers. Mechanical considerations - model systems - phenomenological theories of relaxation and viscosity. In First report on viscosity and plasticity, chapter 1, pages 5-67. Nordemann Publishing, New York, 1939.

[11] R. Bustamante. Some topics on a new class of elastic bodies. Proc. R. Soc. Lond. Ser. A Math. Phys. Eng. Sci., 465(2105):1377-1392, 2009.

[12] R. Bustamante and K.R. Rajagopal. A note on plane strain and plane stress problems for a new class of elastic bodies. Mathematics and Mechanics of Solids, 15(2):229-238, 2010.

[13] R. Bustamante and K.R. Rajagopal. Solutions of some simple boundary value problems within the context of a new class of elastic materials. International Journal of Non-Linear Mechanics, 46:376-386, 2011. 
[14] R. Bustamante and K.R. Rajagopal. On the inhomogeneous shearing of a new class of elastic bodies. Mathematics and Mechanics of Solids, 17(7):762-778, 2012.

[15] M.M. Carroll. Must elastic materials be hyperelastic? Mathematics and Mechanics of Solids, 14(4):369-376, 2009.

[16] A.L. Cauchy. Recherches sur l'équilibre et le mouvement intérieur des corps solides ou fluides, elastiques ou non élastiques. Bull. Soc. Philomath., 1823-1824:9-13, 1823.

[17] A.L. Cauchy. Sur les équations qui experiments les conditions d'équilibre ou le lois du mouvement intérieur, d'un corps solide, élastique ou non élastique. Ex. de Math., 3:160, 1828.

[18] B.D. Coleman and W. Noll. An approximation theorem for functionals, with applications in continuum mechanics. Arch. Ration. Mech. Anal., 6:355-370, 1960.

[19] S. Darbha, K.B. Nakshatrala, and K.R. Rajagopal. On the vibrations of lumped parameter systems governed by differential-algebraic equations. Journal of the Franklin Institute, 347(1):87-101, 2010. Dynamics and Control.

[20] L. Diening, C. Kreuzer, and E. Süli. Finite element approximation of steady flows of incompressible fluids with implicit power-law-like rheology. SIAM J. Numer. Anal., 51(2):984-1015, 2013.

[21] J.E. Dunn and K.R. Rajagopal. Fluids of differential type: Critical review and thermodynamic analysis. Int. J. Engng. Sci., 33:689-729, 1995.

[22] A.D. Freed. Soft Solids - A primer to the theoretical mechanics of materials. Create Space Independent Publishing Platform, Norh Charleston, South Carolina, 2012.

[23] A.D. Freed and D.R. Einstein. An implicit elastic theory for lung parenchyma. Internat. J. Engrg. Sci., 62:3147, 2013.

[24] K. Gou, M. Mallikarjuna, K.R. Rajagopal, and J.R. Walton. Modeling fracture in the context of a strainlimiting theory of elasticity: A single plane-strain crack. International Journal of Engineering Science, in press, 2014. DOI: 10.1016/j.ijengsci.2014.04.018.

[25] G. Green. On the Laws of the Reflexion and Refraction of Light at the common Surface of two non-crystallized Media. Trans. Cambr. Phil. Soc., VII(I):1-24, 1839.

[26] G. Green. On the Propagation of Light in Crystallized Media. Trans. Cambr. Phil. Soc., VII(II):121-140, 1841.

[27] D. Hooke. De Potentia Resistutiva. In R.T. Gunther, editor, The Cutler Lectures of Robert Hooke, volume VIII of Early Science in Oxford, pages 331-356. Oxford University Press, Oxford, 1931.

[28] M. Kambapalli, K. Kannan, and K.R. Rajagopal. Circumferential stress waves in a non-linear cylindrical annulus in a new class of elastic materials. The Quarterly Journal of Mechanics and Applied Mathematics, 67(2):193-203, 2014

[29] K. Kannan, K.R. Rajagopal, and G. Saccomandi. Unsteady motions of a new class of elastic solids. To appear in Wave Motion, 2014.

[30] S. Karra, V. Průša, and K.R. Rajagopal. On Maxwell fluids with relaxation time and viscosity depending on the pressure. Int. J. Non-Linear Mech., 46(6):819-827, 2011.

[31] J.K. Knowles and E. Sternberg. An asymptotic finite-deformation analysis of the elastostatic field near the tip of a crack. Journal of Elasticity, 3(2):67-107, 1973.

[32] V. Kulvait, J. Málek, and K.R. Rajagopal. Anti-plane stress state of a plate with a V-notch for a new class of elastic solids. Int. J. Fract., 179(1-2):59-73, 2013.

[33] T. Li, J.W. Jr.. Morris, N. Nagasako, S. Kuramoto, and D.C. Chrzan. "Ideal" engineering alloys. Phys. Rev. Lett., 98:105503, 2007.

[34] J. Málek, J. Nečas, M. Rokyta, and M. Růžička. Weak and measure-valued solutions to evolutionary PDEs. Chapman \& Hall, London, 1996.

[35] J.C. Maxwell. On the dynamical theory of gases. Philos. Trans. R. Soc., 157:49-88, 1867.

[36] J.E. McKinney and H.V. Belcher. Dynamic compressibility of polyvinylacetate and its relation to free volume. J. Res. Nat. Bur. Stand. Sect. A. Phys. Chem. A, 67:43-53, 1963.

[37] C.L.M.H. Navier. Memoire sur le lois de l'equilibre at du mouvement des corps solideselastiques. Mem. Acad. Sci. Inst. France, 7:375-393, 1821.

[38] C.L.M.H. Navier. Sur les lois du mouvement des fluids, en ayanterpard a l'adhesion des molecules. Annalen der Chemie, 19:244-260, 1821.

[39] J.G. Oldroyd. On the formulation of rheological equations of state. Proc. R. Soc. A-Math. Phys. Eng. Sci., 200(1063):523-541, 1950.

[40] A. Ortiz, R. Bustamante, and K.R. Rajagopal. A numerical study of a plate with a hole for a new class of elastic bodies. Acta Mech., 223(9):1971-1981, 2012.

[41] S.D. Poisson. Mémorie l'équilibre et du mouvement des corps solides élastiques. Mem. Acad. Sci. Inst. France, 8:357-570, 1828.

[42] S.D. Poisson. Mémorie sur les Équations générales de l'équilibre et du mouvements des Corps solides, élastiques et fluides. Journal de l'École Polytechnique, 13:1-174, 1831.

[43] L. Prandtl. Spannungsverteilung in plastischen körpen. In Proceeding of the First Int. Congr. Appl. Mech., pages 43-54, Delft, 1924.

[44] D. Pražák and K.R. Rajagopal. Mechanical oscillators described by a system of differential-algebraic equations. Applications of Mathematics, 57(2):129-142, 2012.

[45] V. Průša and K.R. Rajagopal. On implicit constitutive relations for materials with fading memory. J. NonNewton. Fluid Mech., 181-182:22-29, 2012. 
[46] K.R. Rajagopal. On implicit constitutive theories. Appl. Math., 48:279-319, 2003.

[47] K.R. Rajagopal. On implicit constitutive theories for fluids. J. Fluid Mech., 550:243-249, 2006.

[48] K.R. Rajagopal. Elasticity of elasticity. Z. Angew. Math. Phys., 58:309-417, 2007.

[49] K.R. Rajagopal. A note on a reappraisal and generalization of the Kelvin-Voigt model. Mechanics Research Communications, 36(2):232-235, 2009.

[50] K.R. Rajagopal. A generalized framework for studying the vibrations of lumped parameter systems. Mechanics Research Communications, 37(5):463-466, 2010.

[51] K.R. Rajagopal. Conspectus of concepts of elasticity. Math. Mech. Solids, 16(5):536-562, 2011.

[52] K.R. Rajagopal. Non-linear elastic bodies exhibiting limiting small strain. Math. Mech. Solids, 16:122-139, 2011.

[53] K.R. Rajagopal. On a new class of models in elasticity. J. Math. Comp. Appl., 15:506-528, 2011.

[54] K.R. Rajagopal. A note on material symmetry for bodies defined by implicit constitutive equations. Submitted to Mechanics Reserach Communications, 2014.

[55] K.R. Rajagopal. On the nonlinear elastic response of bodies in the small strain range. Acta Mech., 225:15451553,2014

[56] K.R. Rajagopal and G. Saccomandi. The mechanics and mathematics of the effect of pressure on the shear modulus of elastomers. Proc. R. Soc. A, 465:3859-3874, 2009.

[57] K.R. Rajagopal and U. Saravanan. Spherical inflation of a class of compressible elastic bodies. International Journal of Non-Linear Mechanics, 46(9):1167-1176, 2011.

[58] K.R. Rajagopal and A.R. Srinivasa. On the response of non-dissipative solids. Proc. R. Soc. Lond. Ser. A Math. Phys. Eng. Sci., 463(2078):357-367, 2007.

[59] K.R. Rajagopal and A.R. Srinivasa. On a class of non-dissipative materials that are not hyperelastic. Proc. $R$. Soc. Lond. Ser. A Math. Phys. Eng. Sci., 465(2102):493-500, 2009.

[60] K.R. Rajagopal and J. Walton. Modeling fracture in the context of strain-limiting theory of elasticity. Int. J. Fract., 169:39-48, 2011.

[61] A. Reuss. Berücksichtigung der elastischen Formänderung in der Plastizitätstheorie. Z. Angenew. Math. Mech., 10:266-271, 1930.

[62] R.S. Rivlin. Second and higher order theories for the flow of a viscoelastic fluid in a non-circular pipe. In M. Reiner, editor, Second-Order Effects in Elasticity, Plasticity, and Fluid Dynamics, volume I, pages 668677. Macmillan, New York, 1964.

[63] R.S. Rivlin and J.L. Ericksen. Stress-deformation relations for isotropic materials. J. Ration. Mech. Anal., 4:323-425, 1955.

[64] J.C. Saint-Venant. Not a joindre au Memoire sur la dynamique des fluids. Comptes Rendus, 17:1240-1224, 1843.

[65] T. Saito, T. Furuta, J.H. Hwang, S. Kuramoto, K. Nishino, N. Suzuki, R. Chen, A. Yamada, K. Ito, Y. Seno, T. Nonaka, H. Ikehata, N. Nagasako, C. Iwamoto, Y. Ikuhara, and T. Sakuma. Multifunctional alloys obtained via a dislocation-free plastic deformation mechanism. Science, 300:464-467, 2003.

[66] H. Singh and A.W. Nolle. Pressure Dependence of the Viscoelastic Behavior of Polyisobutylene. J. Appl. Phys., 30(3):337-341, 2012.

[67] A.J.M. Spencer. Theory of invariants. In A.C. Eringen, editor, Continuum Physics, volume I. Academic Press, New York, 1971.

[68] G.G. Stokes. On the theories of internal friction of fluids in motion, and of the equilibrium and motion of elastic solids. Trans. Camb. Phil. Soc., 8:287-341, 1845.

[69] A.Z. Szeri. Fluid film lubrication: theory and design. Cambridge University Press, Cambridge, 2011.

[70] R.J. Talling, R.J. Dashwood, M. Jackson, S. Kuramoto, and D. Dye. Determination of $\left(c_{11}-c_{12}\right)$ in Ti-36Nb2Ta-3Zr-0.3O (wt.\%) (gum metal). Scr. Mater., 59:669-672, 2008.

[71] A. M. Tarantino. Nonlinear fracture mechanics for an elastic Bell material. Quart. J. Mech. Appl. Math., 50:436-456, 1997.

[72] L. Todhunter. A history of the theory of elasticity and strength of materials. Cambridge University Press, Cambridge, 1886.

[73] C. Truesdell and H. Moon. Inequalities sufficient to ensure semi-invertibility of isotropic functions. J. Elast., 5:183-189, 1975.

[74] E. Withey, M. Jin, A. Minor, S. Kuramoto, D.C. Chrzan, and J.W. Jr.. Morris. The deformation of "Gum Metal" in nanoindentation. Mater. Sci. Eng. A, 493:26-32, 2008.

[75] S.Q. Zhang, S.J. Li, M.T. Jia, Y.L. Hao, and R. Yang. Fatigue properties of a multifunctional titanium alloy exhibiting nonlinear elastic deformation behavior. Scr. Mater., 60:733-736, 2009. 


\section{Appendix A. Proofs of the auxiliary Results}

Proof of Lemma 4.1. We prove here only (56). The other inequalities can be established in the same way. Clearly,

$$
\begin{aligned}
(\mu & \left.\left(\left|\mathbf{W}_{1}\right|\right) \mathbf{W}_{1}-\mu\left(\left|\mathbf{W}_{2}\right|\right) \mathbf{W}_{2}\right) \cdot\left(\mathbf{W}_{1}-\mathbf{W}_{2}\right) \\
= & \left(\mathbf{W}_{1}-\mathbf{W}_{2}\right) \cdot \int_{0}^{1} \frac{\mathrm{d}}{\mathrm{d} s}\left(\mu\left(\left|\mathbf{W}_{2}+s\left(\mathbf{W}_{1}-\mathbf{W}_{2}\right)\right|\right)\left(\mathbf{W}_{2}+s\left(\mathbf{W}_{1}-\mathbf{W}_{2}\right)\right) \mathrm{d} s\right. \\
= & \int_{0}^{1} \mu\left(\left|\mathbf{W}_{2}+s\left(\mathbf{W}_{1}-\mathbf{W}_{2}\right)\right|\right)\left|\mathbf{W}_{1}-\mathbf{W}_{2}\right|^{2} \mathrm{~d} s \\
& +\int_{0}^{1} \frac{\mu^{\prime}\left(\left|\mathbf{W}_{2}+s\left(\mathbf{W}_{1}-\mathbf{W}_{2}\right)\right|\right.}{\left|\mathbf{W}_{2}+s\left(\mathbf{W}_{1}-\mathbf{W}_{2}\right)\right|}\left|\left(\mathbf{W}_{2}+s\left(\mathbf{W}_{1}-\mathbf{W}_{2}\right)\right) \cdot\left(\mathbf{W}_{1}-\mathbf{W}_{2}\right)\right|^{2} \mathrm{~d} s \\
= & : \int_{0}^{1} I(s) \mathrm{d} s .
\end{aligned}
$$

First, in case that $\mu^{\prime}\left(\left|\mathbf{W}_{2}+s\left(\mathbf{W}_{1}-\mathbf{W}_{2}\right)\right|\right) \geq 0$, we use (A2) to deduce that (note that $s \in(0,1)$ )

$$
I(s) \geq \frac{C_{1}\left|\mathbf{W}_{1}-\mathbf{W}_{2}\right|^{2}}{\kappa+\left|\mathbf{W}_{2}+s\left(\mathbf{W}_{1}-\mathbf{W}_{2}\right)\right|} \geq \frac{C_{1}\left|\mathbf{W}_{1}-\mathbf{W}_{2}\right|^{2}}{\kappa+\left|\mathbf{W}_{1}\right|+\left|\mathbf{W}_{2}\right|} \geq \frac{C_{1} \kappa^{\alpha}\left|\mathbf{W}_{1}-\mathbf{W}_{2}\right|^{2}}{\kappa+\left|\mathbf{W}_{1}\right|+\left|\mathbf{W}_{2}\right|} .
$$

If $\mu^{\prime}\left(\left|\mathbf{W}_{2}+s\left(\mathbf{W}_{1}-\mathbf{W}_{2}\right)\right|\right) \leq 0$, then (A4) yields

$$
\begin{aligned}
I(s) & \geq\left|\mathbf{W}_{1}-\mathbf{W}_{2}\right|^{2}\left(\mu\left(\left|\mathbf{W}_{2}+s\left(\mathbf{W}_{1}-\mathbf{W}_{2}\right)\right|\right)+\mu^{\prime}\left(\left|\mathbf{W}_{2}+s\left(\mathbf{W}_{1}-\mathbf{W}_{2}\right)\right|\right)\left|\mathbf{W}_{2}+s\left(\mathbf{W}_{1}-\mathbf{W}_{2}\right)\right|\right) \\
& =\left|\mathbf{W}_{1}-\mathbf{W}_{2}\right|^{2}(\mu(t) t)_{t=\left|\mathbf{W}_{2}+s\left(\mathbf{W}_{1}-\mathbf{W}_{2}\right)\right|}^{\prime} \geq \frac{C_{1}\left|\mathbf{W}_{1}-\mathbf{W}_{2}\right|^{2}}{\left(\kappa+\left|\mathbf{W}_{2}+s\left(\mathbf{W}_{1}-\mathbf{W}_{2}\right)\right|\right)^{1+\alpha}} \\
& \geq \frac{C_{1}\left|\mathbf{W}_{1}-\mathbf{W}_{2}\right|^{2}}{\left(\kappa+\left|\mathbf{W}_{1}\right|+\left|\mathbf{W}_{2}\right|\right)^{1+\alpha}} .
\end{aligned}
$$

Using these inequalities in (138), we deduce (55). Finally, since for any $a \geq b \geq 0$ we have (note that $\alpha \leq 1)$

$$
\begin{aligned}
a^{\frac{1-\alpha}{2}}-b^{\frac{1-\alpha}{2}} & =\frac{\left(a^{\frac{1-\alpha}{2}}-b^{\frac{1-\alpha}{2}}\right)\left(a^{\frac{1+\alpha}{2}}+b^{\frac{1+\alpha}{2}}\right)}{a^{\frac{1+\alpha}{2}}+b^{\frac{1+\alpha}{2}}} \\
& =\frac{a-b+a^{\frac{1-\alpha}{2}} b^{\frac{1+\alpha}{2}}-a^{\frac{1+\alpha}{2}} b^{\frac{1-\alpha}{2}}}{a^{\frac{1+\alpha}{2}}+b^{\frac{1+\alpha}{2}}} \leq \frac{2(a-b)}{a^{\frac{1+\alpha}{2}}+b^{\frac{1+\alpha}{2}}},
\end{aligned}
$$

we thus get that

$$
\begin{aligned}
\left|\left(\kappa+\left|\mathbf{W}_{1}\right|\right)^{\frac{1-\alpha}{2}}-\left(\kappa+\left|\mathbf{W}_{2}\right|\right)^{\frac{1-\alpha}{2}}\right|^{2} & \leq \frac{4\left(\left|\mathbf{W}_{1}\right|-\left|\mathbf{W}_{2}\right|\right)^{2}}{\left(\left(\kappa+\left|\mathbf{W}_{1}\right|\right)^{\frac{1+\alpha}{2}}+\left(\kappa+\left|\mathbf{W}_{2}\right|\right)^{\frac{1+\alpha}{2}}\right)^{2}} \\
& \leq \frac{C\left(\left|\mathbf{W}_{1}-\mathbf{W}_{2}\right|\right)^{2}}{\left(\kappa+\left|\mathbf{W}_{1}\right|+\left|\mathbf{W}_{2}\right|\right)^{1+\alpha}} .
\end{aligned}
$$

By using this inequality in (55), we obtain (56).

Proof of Lemma 4.2. We prove the result for a smooth $\mathbf{T}$; a density argument then yields the desired inequality. For a smooth $\mathbf{T}$, we have, for any $i, j, k=1, \ldots, d$,

$$
D_{k} \mathbf{T}_{i j}=D_{k} \mathbf{T}_{i j}^{d}+\frac{\delta_{i j} D_{k}(\operatorname{tr} \mathbf{T})}{d}
$$

We see that to prove (60) it is enough to rewrite $D_{k}(\operatorname{tr} \mathbf{T})$ in an appropriate form. Hence, for any $i, k$ we have the following expression (where we do not sum with respect to $i$ ):

$$
D_{k} \mathbf{T}_{i i}=D_{k} \mathbf{T}_{i i}^{d}+\frac{D_{k}(\operatorname{tr} \mathbf{T})}{d} .
$$


Summing the above identity with respect to $i$ over all $i \neq k$, we get (we again do not sum with respect to $k$ )

$$
D_{k}(\operatorname{tr} \mathbf{T})-D_{k} \mathbf{T}_{k k}=\sum_{i: i \neq k} D_{k} \mathbf{T}_{i i}=\sum_{i: i \neq k} D_{k} \mathbf{T}_{i i}^{d}+\frac{d-1}{d} D_{k}(\operatorname{tr} \mathbf{T})
$$

Consequently, after a simple algebraic manipulation we have

$$
\begin{aligned}
\frac{D_{k}(\operatorname{tr} \mathbf{T})}{d} & =\sum_{i: i \neq k} D_{k} \mathbf{T}_{i i}^{d}+D_{k} \mathbf{T}_{k k}=\sum_{i: i \neq k} D_{k} \mathbf{T}_{i i}^{d}+\sum_{i=1}^{d} D_{i} \mathbf{T}_{i k}-\sum_{i: i \neq k} D_{i} \mathbf{T}_{i k} \\
& =\sum_{i: i \neq k} D_{k} \mathbf{T}_{i i}^{d}+(\operatorname{div} \mathbf{T})_{k}-\sum_{i: i \neq k} D_{i} \mathbf{T}_{i k}^{d} .
\end{aligned}
$$

Direct substitution of the corresponding term into (139) leads to

$$
D_{k} \mathbf{T}_{i j}=D_{k} \mathbf{T}_{i j}^{d}+\delta_{i j} \sum_{l: l \neq k} D_{k} \mathbf{T}_{l l}^{d}-D_{l} \mathbf{T}_{l k}^{d}+\delta_{i j}(\operatorname{div} \mathbf{T})_{k}
$$

and (60) follows.

To prove (59), we first observe that

$$
\begin{aligned}
\|\mathbf{T}\|_{p}^{p}= & \int_{\Omega} \mathbf{T} \cdot\left(\mathbf{T}|\mathbf{T}|^{p-2}-\frac{1}{|\Omega|} \int_{\Omega} \mathbf{T}|\mathbf{T}|^{p-2} \mathrm{~d} y\right) \mathrm{d} x \\
& \quad+\int_{\Omega} \mathbf{T} \cdot\left(\frac{1}{|\Omega|} \int_{\Omega} \mathbf{T}|\mathbf{T}|^{p-2} \mathrm{~d} y\right) \mathrm{d} x \\
\leq & \int_{\Omega} \mathbf{T} \cdot\left(\mathbf{T}|\mathbf{T}|^{p-2}-\frac{1}{|\Omega|} \int_{\Omega} \mathbf{T}|\mathbf{T}|^{p-2} \mathrm{~d} y\right) \mathrm{d} x+C(\Omega, p)\|\mathbf{T}\|_{p}^{p-1}\left|\int_{\Omega} \mathbf{T} \mathrm{d} x\right|,
\end{aligned}
$$

where for the second inequality we used the Hölder inequality. Consequently,

$$
\|\mathbf{T}\|_{p}^{p} \leq C \int_{\Omega} \mathbf{T} \cdot\left(\mathbf{T}|\mathbf{T}|^{p-2}-\frac{1}{|\Omega|} \int_{\Omega} \mathbf{T}|\mathbf{T}|^{p-2} \mathrm{~d} y\right) \mathrm{d} x+C\left|\int_{\Omega} \mathbf{T} \mathrm{d} x\right|^{p} .
$$

Next, using the Bogovskiĭ lemma we can find $G^{k} \in W_{0}^{1, p^{\prime}}(\Omega)^{d \times d}$ for $k=1, \ldots, d$ such that

$$
\begin{aligned}
\sum_{k=1}^{d} D_{k} \mathbf{G}^{k} & =\mathbf{T}|\mathbf{T}|^{p-2}-\frac{1}{|\Omega|} \int_{\Omega} \mathbf{T}|\mathbf{T}|^{p-2} \mathrm{~d} y \quad \text { in } \Omega, \\
\left\|\mathbf{G}^{k}\right\|_{1, p^{\prime}} & \leq C\left\|\mathbf{T}|\mathbf{T}|^{p-2}-\frac{1}{|\Omega|} \int_{\Omega} \mathbf{T}|\mathbf{T}|^{p-2} \mathrm{~d} y\right\|_{p^{\prime}} \leq C\|\mathbf{T}\|_{p}^{p-1} .
\end{aligned}
$$


Then, using the definition of $\mathbf{G}^{k}$, integration by parts (note that $\mathbf{G}^{k}$ vanishes on $\partial \Omega$ ), the identity (140) and Hölder's inequality, we get

$$
\begin{aligned}
\int_{\Omega} \mathbf{T} \cdot\left(\mathbf{T}|\mathbf{T}|^{p-2}-\frac{1}{|\Omega|} \int_{\Omega} \mathbf{T}|\mathbf{T}|^{p-2} \mathrm{~d} y\right) \mathrm{d} x=\sum_{i, j, k=1}^{d} \int_{\Omega} \mathbf{T}_{i j} D_{k} \mathbf{G}_{i j}^{k} \mathrm{~d} x \\
=-\sum_{i, j, k=1}^{d} \int_{\Omega} D_{k} \mathbf{T}_{i j} \mathbf{G}_{i j}^{k} \mathrm{~d} x \\
=-\sum_{i, j, k=1}^{d} \int_{\Omega}\left(D_{k} \mathbf{T}_{i j}^{d}+\delta_{i j} \sum_{l ; l \neq k} D_{k} \mathbf{T}_{l l}^{d}-D_{l} \mathbf{T}_{l k}^{d}+\delta_{i j}(\operatorname{div} \mathbf{T})_{k}\right) \mathbf{G}_{i j}^{k} \mathrm{~d} x \\
=\int_{\Omega} \sum_{k=1}^{d} \mathbf{T}^{d} \cdot D_{k} \mathbf{G}^{k}+\sum_{k, l ; k \neq l}\left(\mathbf{T}_{l l}^{d} D_{k}\left(\operatorname{tr} \mathbf{G}^{k}\right)-\mathbf{T}_{l k}^{d} D_{l}\left(\operatorname{tr} \mathbf{G}^{k}\right)\right) \\
\quad+\sum_{i, k=1}^{d} \mathbf{T}_{i k} D_{i} \operatorname{tr}\left(\mathbf{G}^{k}\right) \mathrm{d} x \\
\leq C \int_{\Omega}\left|\mathbf{T}^{d}\|\nabla \mathbf{G} \mid+\| \mathbf{G}^{k} \|_{1, p^{\prime}} \sup _{\boldsymbol{w} \in W_{0}^{1, p^{\prime}}(\Omega)^{d} ;\|\boldsymbol{w}\|_{1, p} \leq 1} \int_{\Omega} \mathbf{T} \cdot \nabla \boldsymbol{w} \mathrm{d} x\right. \\
\leq C \mid \mathbf{T}_{p}^{p-1}\left(\left\|\mathbf{T}^{d}\right\|_{p}+\sup _{\boldsymbol{w} \in W_{0}^{1, p^{\prime}}(\Omega)^{d} ;\|\boldsymbol{w}\|_{1, p} \leq 1} \int_{\Omega} \mathbf{T} \cdot \nabla \boldsymbol{w} \mathrm{d} x\right)
\end{aligned}
$$

Finally, substituting this into (141) and using the simple inequality

$$
\left|\int_{\Omega} \mathbf{T} \mathrm{d} x\right| \leq\left|\int_{\Omega} \mathbf{T}^{d} \mathrm{~d} x\right|+\left|\int_{\Omega} \operatorname{tr} \mathbf{T} \mathrm{d} x\right| \leq C\left\|\mathbf{T}^{d}\right\|_{p}+\left|\int_{\Omega} \operatorname{tr} \mathbf{T} \mathrm{d} x\right|
$$

we get (59). Thus, the proof is complete.

Proof of Lemma 4.3. Assume that $\boldsymbol{u} \in W_{0}^{1,1}(\Omega)^{d}$ with $\boldsymbol{\varepsilon}(\boldsymbol{u}) \in L^{\infty}(\Omega)^{d \times d}$ is arbitrary; extending $\boldsymbol{u}$ by zero outside $\Omega$, we get $\boldsymbol{u} \in W^{1, p}\left(\mathbb{R}^{d}\right)^{d}$ with $\boldsymbol{\varepsilon}(\boldsymbol{u}) \in L^{\infty}\left(\mathbb{R}^{d}\right)^{d \times d}$. Then, by an application of Korn's inequality, we deduce that $\boldsymbol{u} \in W_{0}^{1, p}(\Omega)^{d}$ for all $p \in[1, \infty)$. Since $\Omega \in \mathcal{C}$, we have (thanks to the definition of a domain with continuous boundary) that there exist positive constants $\tilde{\alpha}, \tilde{\beta}>0$, continuous functions $a^{\nu}: \mathbb{R}^{d-1} \rightarrow \mathbb{R}$, orthogonal matrices $\mathbf{Q}^{\nu}$ (by orthogonal, we mean that $\mathbf{Q Q}^{\mathrm{T}}=\mathbf{I}$ ), points $\boldsymbol{x}_{0}^{\nu}$ with $\nu=1, \ldots, k$, where $k \in \mathbb{N}$, and $k$-coordinate systems given by $\boldsymbol{x}^{\nu}:=\mathbf{Q}^{\nu}\left(\boldsymbol{x}-\boldsymbol{x}_{0}^{\nu}\right)$ such that, upon denoting $P \boldsymbol{x}^{\nu}:=\left(x_{1}^{\nu}, \ldots, x_{d-1}^{\nu}\right)$, we have that

$$
\begin{aligned}
\tilde{\Omega}_{+}^{\nu} & :=\left\{\boldsymbol{x} \in \mathbb{R}^{d} ;\left|P \boldsymbol{x}^{\nu}\right|<\tilde{\alpha}, a^{\nu}\left(P \boldsymbol{x}^{\nu}\right)<x_{d}^{\nu}<a^{\nu}\left(P \boldsymbol{x}^{\nu}\right)+\tilde{\beta}\right\} & & \subset \Omega, \\
\tilde{\Omega}_{-}^{\nu} & :=\left\{\boldsymbol{x} \in \mathbb{R}^{d} ;\left|P \boldsymbol{x}^{\nu}\right|<\tilde{\alpha}, a^{\nu}\left(P \boldsymbol{x}^{\nu}\right)-\tilde{\beta}<x_{d}^{\nu}<a^{\nu}\left(P \boldsymbol{x}^{\nu}\right)\right\} & & \subset \mathbb{R}^{d} \backslash \bar{\Omega}, \\
\partial \tilde{\Omega}^{\nu} & :=\left\{\boldsymbol{x} \in \mathbb{R}^{d} ;\left|P \boldsymbol{x}^{\nu}\right|<\tilde{\alpha}, a^{\nu}\left(P \boldsymbol{x}^{\nu}\right)=x_{d}^{\nu}\right\} & & \subset \partial \Omega,
\end{aligned}
$$

and we have a covering of the boundary, i.e.,

$$
\bigcup_{\nu=1}^{k} \partial \tilde{\Omega}^{\nu}=\partial \Omega
$$

Next, since $\partial \tilde{\Omega}^{\nu}$ is an open (in the sense of $(d-1)$-dimensional topology on $\partial \Omega$ ) covering of a compact set, we can decrease such a covering. More precisely, there exist $\alpha \in(0, \tilde{\alpha})$ and $\beta \in(0, \tilde{\beta})$ for which we can define

$$
\begin{array}{rlrl}
\Omega_{+}^{\nu} & :=\left\{\boldsymbol{x} \in \mathbb{R}^{d} ;\left|P \boldsymbol{x}^{\nu}\right|<\alpha, a^{\nu}\left(P \boldsymbol{x}^{\nu}\right)<x_{d}^{\nu}<a^{\nu}\left(P \boldsymbol{x}^{\nu}\right)+\beta\right\} & \subset \Omega \\
\Omega_{-}^{\nu}:=\left\{\boldsymbol{x} \in \mathbb{R}^{d} ;\left|P \boldsymbol{x}^{\nu}\right|<\alpha, a^{\nu}\left(P \boldsymbol{x}^{\nu}\right)-\beta<x_{d}^{\nu}<a^{\nu}\left(P \boldsymbol{x}^{\nu}\right)\right\} & \subset \mathbb{R}^{d} \backslash \bar{\Omega} \\
\partial \Omega^{\nu}:=\left\{\boldsymbol{x} \in \mathbb{R}^{d} ;\left|P \boldsymbol{x}^{\nu}\right|<\alpha, a^{\nu}\left(P \boldsymbol{x}^{\nu}\right)=x_{d}^{\nu}\right\} & \subset \partial \Omega
\end{array}
$$


and we still obtain

$$
\bigcup_{\nu=1}^{k} \partial \Omega^{\nu}=\partial \Omega .
$$

Thus, by defining the open sets $\Omega^{\nu}:=\Omega_{+}^{\nu} \cup \Omega_{-}^{\nu} \cup \partial \Omega^{\nu}$, we obtain $\partial \Omega \subset \bigcup_{\nu=1}^{k} \Omega^{\nu}$. Finally, it is evident that we can find an open set $\Omega^{k+1} \subset \bar{\Omega}^{k+1} \subset \Omega$ such that

$$
\bar{\Omega} \subset \bigcup_{\nu=1}^{k+1} \Omega^{\nu} .
$$

Hence having such a covering we can construct, for $\nu=1, \ldots, k+1$, nonnegative functions $\tau^{\nu} \in$ $\mathcal{D}\left(\Omega^{\nu}\right)$ such that for all $x \in \bar{\Omega}$ we have

$$
\sum_{\nu=1}^{k+1} \tau^{\nu}(x)=1
$$

Having introduced the necessary technical notations, we continue by defining

$$
\boldsymbol{u}^{\nu}(\boldsymbol{x}):=\boldsymbol{u}(\boldsymbol{x}) \tau^{\nu}(\boldsymbol{x})
$$

It directly follows from the above definition, the fact that $\boldsymbol{u}^{\nu} \equiv \mathbf{0}$ outside $\Omega$ and from (152) that

$$
\sum_{\nu=1}^{k+1} \boldsymbol{u}^{\nu}(\boldsymbol{x})=\boldsymbol{u}(\boldsymbol{x}) \text { in } \mathbb{R}^{d}
$$

Next, for any $\nu=1, \ldots, k$, we define

$$
\boldsymbol{n}^{\nu}:=\left(\mathbf{Q}_{d 1}^{\nu}, \ldots, \mathbf{Q}_{d d}^{\nu}\right)=\left(\mathbf{Q}^{\nu}\right)^{\mathrm{T}}(0, \ldots, 0,1)^{\mathrm{T}}
$$

and for any $1>\delta>0$ and $\nu=1, \ldots, k$ we set

$$
\begin{aligned}
\boldsymbol{u}^{\nu, \delta}(\boldsymbol{x}) & :=\boldsymbol{u}^{\nu}\left(\boldsymbol{x}-\delta \boldsymbol{n}^{\nu}\right), \\
\boldsymbol{u}^{\delta}(\boldsymbol{x}) & :=\sum_{\nu=1}^{k} \boldsymbol{u}^{\nu, \delta}(\boldsymbol{x})+\boldsymbol{u}^{k+1}(\boldsymbol{x}) .
\end{aligned}
$$

Now, for any $p \in[1, \infty)$,

$$
\boldsymbol{u}^{\nu, \delta} \rightarrow \boldsymbol{u}^{\nu} \text { strongly in } W^{1, p}\left(\mathbb{R}^{d}\right)^{d}
$$

and also

$$
\left\|\varepsilon\left(\boldsymbol{u}^{\nu, \delta}\right)\right\|_{\infty} \leq\|\varepsilon(\boldsymbol{u})\|_{\infty}\left\|\tau^{\nu}\right\|_{\infty}+\|\boldsymbol{u}\|_{\infty}\left\|\nabla \tau^{\nu}\right\|_{\infty} \leq C\|\varepsilon(\boldsymbol{u})\|_{\infty}
$$

where the last inequality follows from the Korn inequality (applied in the $W^{1, p}$ norm, for some $p>d$ ) and the embedding theorem. Note that the constant $C$ does not depend on $\delta$.

Finally, we show that $\boldsymbol{u}^{\delta}$ is compactly supported in $\Omega$. First, such a property is trivially true for $\boldsymbol{u}^{k+1}$. Indeed, there exists a $\delta_{0}>0$ such that $\operatorname{dist}(\boldsymbol{x}, \partial \Omega)>\delta_{0}$ for all $\boldsymbol{x} \in \Omega^{k+1}$. Next, since $\boldsymbol{u}^{\nu}$ is supported in $\Omega_{+}^{\nu}$ we deduce that $\boldsymbol{u}^{\nu, \delta}$ is supported in

$$
\Omega_{+}^{\nu, \delta}:=\left\{\boldsymbol{x}:\left(\boldsymbol{x}-\delta \boldsymbol{n}^{\nu}\right) \in \Omega_{+}^{\nu}\right\} .
$$

Our goal is to show that there is a $\delta^{\nu}>0$ such that for all $\boldsymbol{x} \in \Omega_{+}^{\nu, \delta}$ we have $B_{\delta^{\nu}}(\boldsymbol{x}) \subset \Omega$. Clearly, to ensure such a property it suffices to show that $B_{\delta^{\nu}}(\boldsymbol{x}) \subset \tilde{\Omega}_{+}^{\nu}$. Hence, let $\boldsymbol{z} \in \mathbb{R}^{d}$ be arbitrary with $|\boldsymbol{z}| \leq 1$. We then need to show that $\boldsymbol{x}+\delta^{\nu} \boldsymbol{z} \in \tilde{\Omega}_{+}^{\nu}$. Thus, denoting $\boldsymbol{x}^{\nu}=\mathbf{Q}^{\nu}\left(\boldsymbol{x}-\boldsymbol{x}_{0}^{\nu}\right)$ and $\boldsymbol{z}^{\nu}:=\mathbf{Q}^{\nu} \boldsymbol{z}$, we see that it is equivalent to show that

$$
\begin{aligned}
\left|P\left(\boldsymbol{x}^{\nu}+\delta^{\nu} \boldsymbol{z}^{\nu}\right)\right| & <\tilde{\alpha}, \\
a^{\nu}\left(P\left(\boldsymbol{x}^{\nu}+\delta^{\nu} \boldsymbol{z}^{\nu}\right)\right) & <x_{d}^{\nu}+\delta^{\nu} z_{d}^{\nu}<a^{\nu}\left(P\left(\boldsymbol{x}^{\nu}+\delta^{\nu} \boldsymbol{z}^{\nu}\right)\right)+\tilde{\beta} .
\end{aligned}
$$

On the other hand, it follows from the definition of $\Omega_{+}^{\nu, \delta}$ that

$$
\begin{aligned}
\left|P\left(\boldsymbol{x}^{\nu}\right)\right| & <\alpha, \\
a^{\nu}\left(P\left(\boldsymbol{x}^{\nu}\right)\right) & <x_{d}^{\nu}-\delta<a^{\nu}\left(P\left(\boldsymbol{x}^{\nu}\right)\right)+\beta .
\end{aligned}
$$


Hence, we first use (158) to check (156). By the triangle inequality and the fact that $|\boldsymbol{z}|=\left|\boldsymbol{z}^{\nu}\right| \leq 1$ we get

$$
\left|P\left(\boldsymbol{x}^{\nu}+\delta^{\nu} \boldsymbol{z}^{\nu}\right)\right| \leq\left|P\left(\boldsymbol{x}^{\nu}\right)\right|+\left|P\left(\delta^{\nu} \boldsymbol{z}^{\nu}\right)\right| \leq \alpha+\delta^{\nu}
$$

and to guarantee (156) it is enough to assume that

$$
0<\delta^{\nu}<\tilde{\alpha}-\alpha
$$

Similarly for the first inequality in (156) we use (158) to obtain

$$
\begin{aligned}
a^{\nu}\left(P\left(\boldsymbol{x}^{\nu}+\delta^{\nu} \boldsymbol{z}^{\nu}\right)\right) & <x_{d}^{\nu}+\delta^{\nu} z_{d}^{\nu}-\delta^{\nu} z_{d}^{\nu}+a^{\nu}\left(P\left(\boldsymbol{x}^{\nu}+\delta^{\nu} \boldsymbol{z}^{\nu}\right)\right)-a^{\nu}\left(P\left(\boldsymbol{x}^{\nu}\right)\right)-\delta \\
& \leq x_{d}^{\nu}+\delta^{\nu} z_{d}^{\nu}+\left|a^{\nu}\left(P\left(\boldsymbol{x}^{\nu}+\delta^{\nu} \boldsymbol{z}^{\nu}\right)\right)-a^{\nu}\left(P\left(\boldsymbol{x}^{\nu}\right)\right)\right|+\delta^{\nu}-\delta,
\end{aligned}
$$

and to get (156) it is enough to select $\delta^{\nu}$ such that

$$
\left|a^{\nu}\left(P\left(\boldsymbol{x}^{\nu}+\delta^{\nu} \boldsymbol{z}^{\nu}\right)\right)-a^{\nu}\left(P\left(\boldsymbol{x}^{\nu}\right)\right)\right|+\delta^{\nu} \leq \delta .
$$

Similarly, we also have

$$
\begin{aligned}
x_{d}^{\nu}+\delta^{\nu} z_{d}^{\nu} & <a^{\nu}\left(P\left(\boldsymbol{x}^{\nu}\right)\right)+\delta+\delta^{\nu} z_{d}^{\nu}+\beta \\
& \leq a^{\nu}\left(P\left(\boldsymbol{x}^{\nu}+\delta^{\nu} \boldsymbol{z}^{\nu}\right)\right)+\left|a^{\nu}\left(P\left(\boldsymbol{x}^{\nu}+\delta^{\nu} \boldsymbol{z}^{\nu}\right)\right)-a^{\nu}\left(P\left(\boldsymbol{x}^{\nu}\right)\right)\right|+\delta^{\nu}+\delta+\beta,
\end{aligned}
$$

and in order to guarantee the second inequality in (157) it is enough to assume that

$$
\left|a^{\nu}\left(P\left(\boldsymbol{x}^{\nu}+\delta^{\nu} \boldsymbol{z}^{\nu}\right)\right)-a^{\nu}\left(P\left(\boldsymbol{x}^{\nu}\right)\right)\right|+\delta^{\nu}+\delta \leq \tilde{\beta}-\beta .
$$

Finally, we specify the choice of $\delta, \delta^{\nu}$ so that (160), (161) and (162) hold. First we assume that

$$
0<\delta^{\nu} \leq \delta<\frac{1}{2} \min \{\tilde{\alpha}-\alpha, \tilde{\beta}-\beta\} .
$$

Then, (160) surely holds. Moreover, we see that if (163) is true, then (161) implies (162). Thus, it remains to check (161). To do so, we see that it suffices to ensure that

$$
\left|a^{\nu}\left(P\left(\boldsymbol{x}^{\nu}+\delta^{\nu} \boldsymbol{z}^{\nu}\right)\right)-a^{\nu}\left(P\left(\boldsymbol{x}^{\nu}\right)\right)\right|+\delta^{\nu} \leq \delta .
$$

Hence, using the uniform continuity of $a$ and the fact that $|\boldsymbol{z}| \leq 1$ we see that for any $\delta>0$ there exists $0 \leq \gamma \leq \delta$ such that for all $\delta^{\nu} \in(0, \gamma)$ the above inequality holds. Thus, having such a $\gamma$ we define the sequence

$$
\boldsymbol{u}^{\gamma}:=\boldsymbol{u}^{\delta} * \varrho_{\gamma},
$$

where $\varrho_{\gamma}$ denotes the standard regularizing kernel of radius $\gamma$. It follows from the above argument that $\boldsymbol{u}^{\gamma} \in \mathcal{D}(\Omega)^{d}$. The rest of the proof is based on standard properties of regularization and we directly obtain the statement of the theorem.

Mathematical Institute, Faculty of Mathematics and Physics, Charles University, Sokolovská 83, 18675 Prague 8, Czech Republic

E-mail address: mbul8060@karlin.mff.cuni.cz

Mathematical Institute, Faculty of Mathematics and Physics, Charles University, Sokolovská 83, 18675 Prague 8, Czech Republic

E-mail address: malek@karlin.mff.cuni.cz

Department of Mechanical Engineering, Texas A\&M University, College Station, TX 77843, USA

E-mail address: krajagopal@tamu.edu

Mathematical Institute, University of Oxford, Woodstock Road, Oxford OX2 6GG, UK

E-mail address: Endre.Suli@maths.ox.ac.uk 